\title{
GEOCHEMISTRY OF GRANULITE FACIES ORTHGNEISSES OF THE JUIZ DE FORA COMPLEX, CENTRAL SEGMENT OF THE RIBEIRA BELT, SOUTHEASTERN BRAZIL
}

\author{
BEATRIZ PASCHOAL DUARTE*, MÁRIO C. H. FIGUEIREDO** (IN MEMORIAN), MÁRIO CAMPOS NETO** \\ \& MONICA HEILBRON*
}

\begin{abstract}
RESUMO GEOQUÍMICA DOS ORTOGNAISSES GRANULÍTICOS DO COMPLEXO JUIZ DE FORA, SEGMENTO CENTRAL DA FAIXA RIBEIRA, BRAZIL O Complexo Juiz de Fora (CJF) foi investigado em sua área-tipo (região de Juiz de Fora, MG) e está inserido em um contexto de evolução geoló gica complexa, policíclica, com o envolvimento de eventos deformacionais e metamórficos de alto grau recorrentes, que se desenvolveram desde o Arqueano até o Neoproterozóico-Neocambriano. O CJF é constituído de ortognaisses, migmatitos e metabasitos na fácies granulito, tendo, subordinadamente, ortognaisses na fácies anfibolito. O mapeamento geológico mostrou que o CJF ocorre como escamas tectônicas interdigitadas a rochas metassedimentares pós-1.8 Ga., com paragêneses da fácies anfibolito, e a paragnaisses migmatíticos com ortopiroxênio. Eventos tectônicos recorrentes obliteraram generalizadamente as feições e a paleogeografia original. O artigo visa investigar as características litogeoquímicas dos ortogranulitos do CJF e elucidar aspectos da sua evolução magmática original e ambiente geotectônico. Dados de campo e petrográficos permitem subdivir estes ortogranulitos em granulitos máficos, intermediários e félsicos. Os elementos imóveis e ETR sugerem que (a) os granulitos máficos sãoo toleiitos de provável evolução a partir de um mesmo magma parental, (b) os intermediários e félsicos são calcioalcalinos e possuem evidências que sugerem relacão petrogenética entre alguns de seus litotipos, e (c) nenhuma relação petrogenética parece existir entre os granulitos máficos e os demais.
\end{abstract}

Palavras-chave: Geoquímica, Granulito, Faixa Ribeira, Precambriano.

\begin{abstract}
The Juiz de Fora Complex (JFC) in the surroundings of Juiz de Fora (MG) town records a complex geological evolution, with recurring deformational and high-grade metamorphic events from the Archean to the Lower Cambrian. The JFC comprises pre-1.8 Ga. granulite facies orthogneisses, migmatites and metabasites, with subordinated amphibolite facies orthogneisses. The JFC occurs as thrust sheets within post-1.8 Ga amphibolite facies metasedimentary rocks and within orthopyroxene-bearing migmatitic paragneisses of undetermined age. Recurring tectonic events led to the general obliteration of original textures, structures and paleogeography. Field and petrographic data allow the subdivision of the orthogranulites into mafic, intermediate, and felsic granulites. Imobile and Rare Earth elements suggest that (a) the mafic granulites are tholeiitic and that each sample represents different stages of differentiation of one parental magma, (b) the intermediate and felsic granulites are calc-alkaline, and (c) no petrogenetic link seems to exist between the tholeiitic and the calc-alkaline rocks, but some evidences point to possible link among the calc-alkaline rocks.
\end{abstract}

Keywords: Geochemistry, Granulite, Ribeira Belt, Precambrian.

INTRODUCTION The terms Juiz de Fora Series (Ebert 1955), Juiz de Fora Gneisses (Cordani et al 1973) belonging to the Paraíba Group (Ebert 1968) and Juiz de Fora Complex (Oliveira 1980, Machado $\mathrm{F}^{\circ}$ et al 1983, Barbosa \& Grossi Sad 1983a, b, c, Grossi Sad \& Barbosa 1985, Pinto 1991, Heilbron 1993, Nogueira 1994, Duarte et al 1994) have always been related to granulite fácies metasediments and /or orthogneisses that crop out along a NE-SW trending belt in southern Minas Gerais State. Geochronological data indicate that these rocks were granulitized during the Transamazonian Event, ca. 2,2 -1,8 Ga. (Delhal et al 1969, Cordani et al. 1973), although the protholiths may be either of Paleoproterozoic (Cordani et al. 1973, Heilbron 1993, Machado et ai 1996, Figueiredo \& Teixeira 1996) or Archean age (Cordani et al. 1973, Machado $\mathrm{F}^{\mathrm{o}}$ et al 1983). A retrogressive metamorphic event, that took place during the Brasiliano Orogeny, ca. 605 - $490 \mathrm{Ma}$. (Delhal et al 1969, Cordani et al. 1973, Valladares 1996, Machado et al 1996), led to partial replacement of the granulite fácies paragenesis by those of the high amphibolite fácies. Figueiredo and Teixeira (1996) re- lated this late metamorphic event to the Rio Doce Orogeny (550-500 Ma.), defined by Campos Neto \& Figueiredo (1992, 1995).

In this work, the Juiz de Fora Complex is defined as composed of pré-1,8 Ga. granulite fácies orthogneisses, migmatites and metabasites, with subordinated lenses of amphibolite facies orthogneisses which occupy part of the central segment of the Neoproterozoic Ribeira mobile belt (Almeida et al. 1973).

GEOLOGICAL SETTING The study area is situated in the surroundings of the Juiz de Fora, Matias Barbosa, Simão Pereira and Belmiro Braga towns, southern Minas Gerais state (Fig. 1). Geological mapping, at 1: 50.000 scale reduced to 1.250.000 (Duarte et al 1994), shows that the JFC occurs as thrust sheets within post-1.8 Ga amphibolite fácies metasedimentary units probably belonging to the Andrelândia Depositional Cycle (Andreis et al. 1989), and orthopyroxene-bearing migmatitic paragneisses of uknown stratigraphic position (Fig. 1).

* Departamento de Geologia Regional e Geotectônica, Faculdade de Geologia, Universidade do Estado do Rio de Janeiro (UERJ), Rua São Francisco Xavier, 524, sala A 4006. Maracanã, Rio de Janeiro, RJ, CEP 20550-900, Telefone: 021 - 587-7102 fax: 021 - 254-6675, Brasil

** Departamento de Geologia Geral, Instituto de Geociências, Universidade de São Paulo (USP), Rua do Lago, 562. Cidade Universitária, São Paulo, SP, CEP 05422-970, Caixa Postal: 11.348, Telefone: 011 - 818-3994 fax: 011 - 818-3993, Brasil 

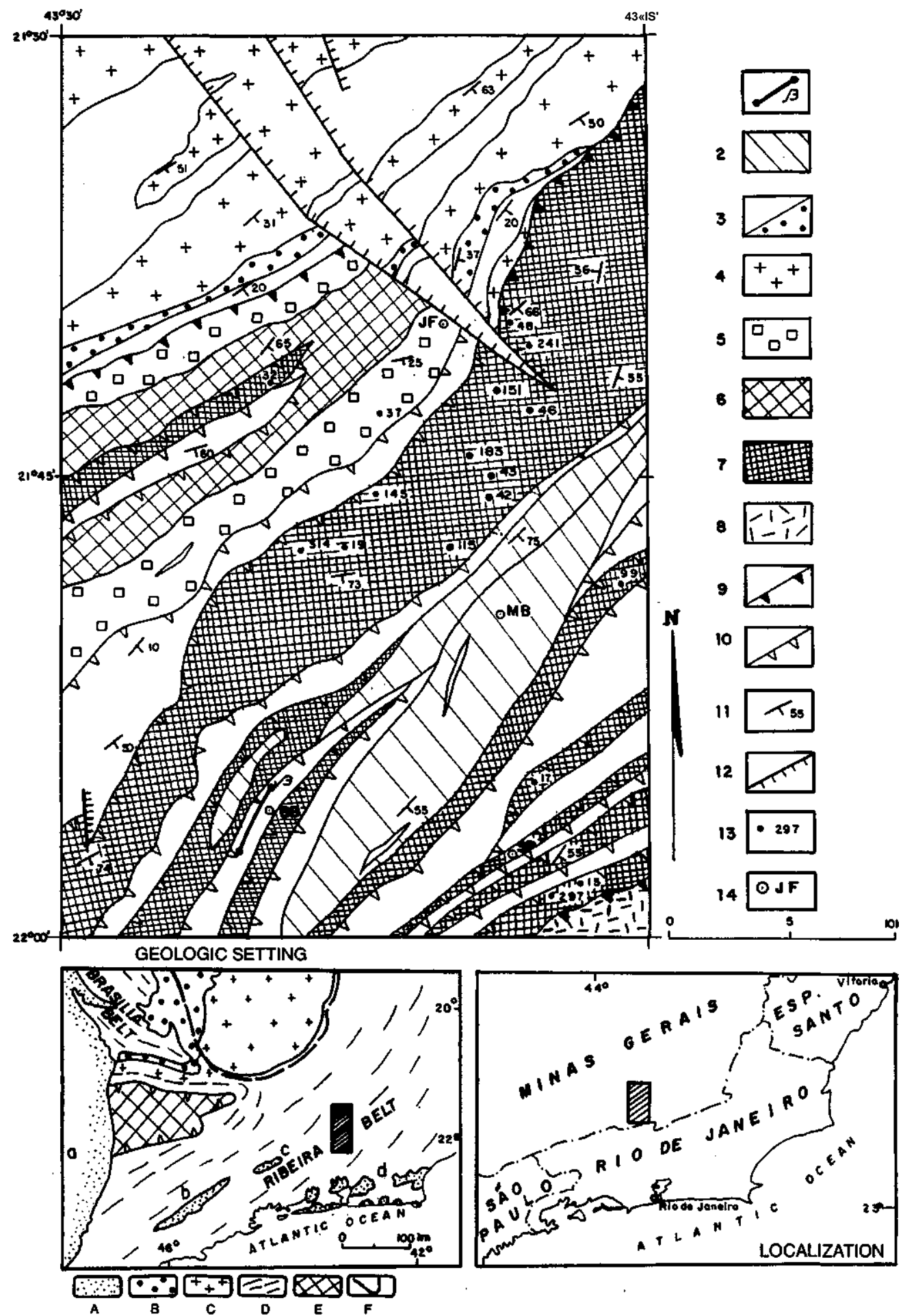

Figure 1- Geologic Map of the Study Area (modified after Duarte et al 1994): 1- Mesozoic mafic dikes; 2 - Neoproterozoic intrusive granitic bodies; 3- Post-1.8 Ga. metasediments (Andrelândia Depositional Cicie and correlated units); 4 - Mantiqueira Complex; 5- Garnet Charnockite; 6 - Opx-bearing paragneisses; 7 - Juiz de Fora Complex (orthogranulites); 8 - Paraíba do Sul Complex; 9 - Main thrust faults; 10 - Thrust faults; 11 - Foliation planes; 12 - Faults with normal component; 13 - Analyzed samples; 14 - Towns (JF - Juiz de Fora; MB - Matias Barbosa; SP - Simão Pereira; BB - Belmiro Braga); A - Phanerozoic Cover (a-Paraná Basin; b-Taubaté Basin; c-Resende Basin; d-Guanabara Rift); B - Bambuí Group; C - San Francisco Craton; D Neoproterozoic Belts; E - Guaxupé Nappe; F - Craton Limits.

Figura 1 - Mapa Geológico da Área Estudada (modificado de Duarte et al. 1994): 1 - Diques básicos Mesozóicos; 2 - Rochas graníticas intrusivas Neoproterozóicas; 3 - Rochas metassedimentares pós-1,8 Ga. (Ciclo Deposicional Andrelândia e correlates); 4 - Complexo Mantiqueira; 5 - Granada Charnockitóide; 6 - Paragnaisses com opx; 7 - Complexo Juiz de Fora (ortogranulitos); 8 - Complexo Paraíba do Sul; 9 - Principais Falhas de Empurrão; 10 - Falhas de Empurrão; 11 - Planos de Foliação; 12 - Falha com componente normal; 13 - Amostras analisadas; 14 - Cidades (JF - Juiz de Fora; MB - Matias Barbosa; SP - Simão Pereira; BB - Belmiro Braga); A - Cebertura Fanerozóica (a-Bacia do Paraná; b-Bacia de Taubaté; c- Bacia de Resende; d-Rifte da Guanabara); B - Grupo Bambuí; C - Cráton do São Francisco; D - Faixas Móveis Neoproterozóicas; E - Nappe de Guaxupé ; F - Limites do Cráton. 
The orthogranulites of the JFC comprise lithotypes of a wide compositional variety. The opx $\pm \mathrm{cpx} \pm$ grt + plg paragenesis indicate metamorphism under granulite fácies. Enderbitic and charno-enderbitic compositions are the most widespread, although mafic and more felsic rocks also occur. These rocks are greenish or caramel and have granoblastic texture. Compositional banding is common and migmatitic structures, when present, contain green leuco-, melano- and paleosomes, suggesting an in situ anatexis before granulitic metamorphism. Granulites become greyish protomylonites, mylonites or ultramylonites near thrust planes of the Brasiliano Orogeny, which in their turn produced retrogessive paragenesis with hydrated minerais such as hornblende and /or biotite at the borders and within pyroxene grains. The new minerais are usually oriented along the younger mylonitic foliation.

FIELD AND PETROGRAPHIC CHARACTERISTICS Fresh exposures of the granulites occur in many quarries and highway and railway cuts, allowing sampling of ali lithotypes. Field and petrographic data classify the lithotypes into three major groups, i.e., mafic, intermediate and felsic granulites.

Mafic Granulites These occur as centimetric bands, lenses, and boudins as well as metric bands within the other groups. They have a dominant granoblastic texture with an $\mathrm{opx}+\mathrm{cpx} \pm \mathrm{hb} \pm$ grt $-\mathrm{f}$ plg paragenesis. Metamorphic retrogressive reactions transformed the pyroxene and plagioclase into hornblende \pm garnet.

\section{Intermediate Granulites MASSIVE ENDER- BITEAND CHARNO-ENDERBITE These rocks are} mainly massive, but may show a weak coarse foliation. Their texture is granoblastic. Protomylonitic and mylonitic textures occur near and along thrust faults. Compositional banding may locally occur due to the intercalation of mafic granulites with enderbites and charno-enderbites. The granulite paragenesis is made up of opx, cpx, plag, ort, and qz, with local retrogressive biotite after pyroxene. Hornblende is absent in these rocks. The associated mafic granulites occur either as centimetric lenses and boudin-shaped enclaves, or as centimetric to metric bands or lenses.

\section{ENDERBITIC GNEISSES AND MIGMATITES}

Unlike

the preceeding group, these rocks have pervasive gneissic banding and mylonitic foliation, and stromatic and shollen migmatitic structures. Hornblende and biotite are common retrogressive phases formed after opx and cpx. Associated mafic granulites occur as centimetric lenses and boudinshaped enclaves.

\section{Felsic Granulites}

CHARNOCKITIC LEUCOSOMES AND GNEISSIC CHARNOCKITES This group is largely dominated by felsic compositions (charnockite and charno-enderbite). These rocks are either massive or slightly foliated. Oriented schlieren is common, suggesting partia! melting during their formation. Petrographically, these lithotypes are characterized by the abundance of biotite retrogressive after orthopyroxene and hornblende, small ammounts of garnet, and by frequent synplectitic intergrowths of biotite and/or hornblende and felsic minerais. Associated mafic granulites occur mainly as small, centimetric bands and lenses. Banding may locally be metric near thrust faults, where it is also more deformed and with mylonitic texture.

INTRUSIVE FELSIC BODIES Felsic bodies commonly show sharp and intrusive contacts with rocks of ali other granulite lithotypes. Two types of felsic intrusive rocks were found and comprise charnockites and quartzose charnockites. The latter, not yet chemically analized, plots within the Ia field of Streckeisen's QAP diagram. The charnockites are médium- grained, hololeucocratic, have few grains of orthopyroxene, and are locally allanite-rich. Mafic granulite were not found in association with this lithotype, although it clearly crosscuts some mafic bands. The quartzose charnockites are coarse grained, essentially composed of quartz and centimetric grains of orthopyroxene, and have enclaves of mafic granulites.

\section{GEOCHEMISTRY AND TECTONIC SETTINGS}

Oliveira $(1980,1982)$ was the first to study the petrology of the JFC rocks and concluded that the mafic granulites have a tholeiitic character, while the intermediate to felsic orthogranulites are of incompatible element-depleted calc-alkaline affinity, being interpreted as residues of crustal melts. Machado $\mathrm{F}^{\mathrm{o}}$ et al (1983) suggest that the tholeiitic and calcalkaline granulites of the JFC are part of an Archean continental or basaltic lower crust. Barbosa \& Grossi Sad (1983c) reached to the same conclusions by mapping and studying these rocks in the eastern Minas Gerais and Rio de Janeiro states. Pinto (1991) suggests that the orthogranulites (mafic and dioritic granulites) were low-K tholeiites, probably of island are or back-arc basins, and that the enderbitic granulites were calc-alkaline rocks. Heilbron (1993), investigating an area SW of Juiz de Fora, proposes that the JFC contains four distinct magmatic groups: (a) mafic tholeiitic granulites with alkaline affinity; (b) mafic tholeiitic granulites; (c) intermediate to felsic calc-alkaline granulites, and (d) intermediate to felsic high-K calc-alkaline granulites. Figueiredo and Teixeira (1996), studying an area north of Juiz de Fora, conclude that the orthogranulites of the JFC derive from (a) a low-K calc-alkaline series and (b) a medium- to high-K calc-alkaline series.

In this study, field data show that the orthogranulites of the JFC are polyciclic and occur as thrust sheets within metasedimentary rocks (Fig. 1). As tectonics and metamorphism obliterated their original textures, structures and paleogeography, the geochemistry of these rocks is an important tool to decifer their original magmatic evolution and tectonic settings.

Field and petrographic data determined the selection of 34 samples of the JFC for chemical analysis. Among these samples, 8 are of mafic granulites, 4 of intermediate massive granulites, 9 of intermediate granulite gneisses and migmatites, 7 of felsic granulites (charnockitic leucosomes and gneissic charnockite), and 7 of intrusive charnockite bodies. The analytical results are shown in Tables 1 to 5. Major, trace, and Rare Earth (REE) elements were determined at the Activation Laboratory (Canada). ICP was used to analyze the major elements and $\mathrm{Sc}, \mathrm{V}, \mathrm{Zr}$, and $\mathrm{Ba}$, while INAA and ICP for the remaining trace elements and the REE.

Major and Trace Element Geochemistry From the chemical classification diagrams of the studied samples (Figs. 2 to 5), it may be concluded that the mafic granulites are gabbros (Fig. 2), the intermediate granulites are quartz 
Table 1 - Chemical composition of the Mafic Granulites. (HMgT - high-Mg tholeiites; MMgT- medium-Mg tholeiites; LMgTlow-Mg tholeiites) (explanation in text). na - not analyzed; $n d$-not detected; (13) - estimated values

Tabela 1-Composição química dos Granulitos Máficos. (HMgT - toleiitos de alto Mg; MMgT - toleiitos de médio Mg; $\mathrm{LMgT}$ - toleiitos de baixo Mg) (explicação no texto), na - não analisado; nd - não detectado; (13) - valores estimados

\begin{tabular}{|c|c|c|c|c|c|c|c|c|}
\hline Sample & $\begin{array}{c}\mathrm{MB}-17 \mathrm{~A} \\
(\mathrm{HMgT})\end{array}$ & $\begin{array}{l}\mathrm{MB}-42 \\
(\mathrm{MMgT})\end{array}$ & $\begin{array}{c}\mathrm{MB}-46 \mathrm{P} \\
(\mathrm{MMgT})\end{array}$ & $\begin{array}{c}\text { MB - 17B } \\
(\mathbf{H M g T})\end{array}$ & $\begin{array}{c}\mathrm{MB} \cdot 15 \mathrm{C} \\
(\mathrm{MMgT})\end{array}$ & $\begin{array}{c}\text { MB - 183A } \\
\text { (LMgT) }\end{array}$ & $\begin{array}{c}\text { MB - 514B } \\
(\mathrm{LMgT})\end{array}$ & $\begin{array}{c}\text { MB - 514C } \\
(\mathrm{LMgT})\end{array}$ \\
\hline $\mathrm{SiO}_{2}$ & 47.21 & 47.62 & 48.67 & 48.77 & 49.21 & 49.41 & 49.72 & 47.8 \\
\hline $\mathrm{TiO}_{2}$ & 0.76 & 1.53 & 0.98 & 0.56 & 1.06 & 1.94 & 2.18 & 2.22 \\
\hline $\mathrm{Al}_{2} \mathrm{O}_{3}$ & 15.3 & 13.29 & 13.69 & 15.12 & 15.22 & 13.85 & 12.86 & 13.27 \\
\hline FeO* & 10.37 & 14.95 & 12.62 & 9.28 & 11.9 & 14.42 & 13.25 & 12.75 \\
\hline $\mathrm{MnO}$ & 0.17 & 0.22 & 0.19 & 0.17 & 0.2 & 0.23 & 0.22 & 0.21 \\
\hline $\mathrm{MgO}$ & 8.85 & 6.48 & 6.43 & 8.93 & 6.68 & 4.74 & 5.32 & 6.19 \\
\hline $\mathrm{CaO}$ & 13.03 & 10.08 & 10.33 & 13.02 & 9.75 & 8.06 & 9.57 & 10.25 \\
\hline $\mathrm{Na}_{2} \mathrm{O}$ & 2.22 & 2.56 & 2.98 & 2.35 & 1.45 & 2.94 & 3.19 & 3.26 \\
\hline $\mathrm{K}_{2} \mathrm{O}$ & 0.66 & 0.38 & 0.83 & 0.46 & 0.58 & 0.74 & 0.61 & 0.88 \\
\hline $\mathrm{P}_{2} \mathrm{O}_{5}$ & 0.04 & 0.15 & 0.08 & 0.06 & 0.12 & 0.22 & 0.31 & 0.22 \\
\hline LOI & 0.45 & nd & 0.05 & 0.37 & 0.85 & 0.7 & 0.68 & 2.02 \\
\hline Total & 100.21 & 98.93 & 98.25 & 100.12 & 98.34 & 98.86 & 99.38 & 100.49 \\
\hline $\mathbf{M g} \#$ & 60.34 & 43.57 & 47.6 & 63.17 & 50.02 & 36.93 & 41.72 & 46.38 \\
\hline $\mathrm{Cr}$ & 120 & na & na & 270 & 140 & na & 100 & 129 \\
\hline $\mathrm{Ni}$ & 146 & 136 & 103 & $13 \mathrm{I}$ & 75 & 61 & 37 & 78 \\
\hline $\mathrm{Co}_{0}$ & 49 & $\mathbf{n a}$ & na & 45 & 48 & na & 43 & 55 \\
\hline $\mathrm{Sc}$ & 43 & 41 & 40 & 42 & 42 & 38 & 40 & 41 \\
\hline V & 226 & 276 & 229 & 194 & 255 & 274 & 267 & 385 \\
\hline $\mathrm{Cu}$ & 109 & 103 & 69 & 20 & 37 & 74 & 78 & 57 \\
\hline $\mathbf{P b}$ & nd & 8 & 16 & nd & nd & 11 & 11 & 12 \\
\hline $\mathrm{Zn}$ & 60 & 112 & 89 & 48 & 96 & 115 & 27 & 28 \\
\hline $\mathbf{R b}$ & 17 & nd & nd & nd & 28 & nd & 3 & 8 \\
\hline $\mathrm{Cs}$ & nd & nd & nd & nd & 0.6 & 2.6 & nd & nd \\
\hline $\mathbf{B a}$ & 45 & 38 & 54 & 24 & 69 & 119 & 150 & 138 \\
\hline $\mathrm{Sr}$ & 116 & 145 & 222 & 94 & 240 & 317 & 264 & 237 \\
\hline $\mathrm{Ta}$ & nd & 0.5 & 0.7 & nd & 0.8 & 0.8 & 1.13 & 0.78 \\
\hline $\mathrm{Nb}$ & na & (8.5) & (11.9) & na & (13.6) & (13.6) & 18.6 & 13.1 \\
\hline Hf & 0.8 & 2 & 1.8 & 0.8 & 1.6 & 3.3 & 5.1 & 3.9 \\
\hline $\mathrm{Zr}$ & 54 & 79 & 63 & 56 & 98 & 129 & 192 & 142 \\
\hline $\mathbf{Y}$ & 20 & 3.1 & 23 & 20 & 38 & 35 & 43 & 32 \\
\hline Th & nd & nd & 0.8 & 0.5 & 0.6 & 0.4 & 1.09 & 1.22 \\
\hline $\mathrm{U}$ & nd & nd & 0.4 & 0.8 & nd & nd & 0.45 & 0.65 \\
\hline La & 1.9 & 5.6 & 6.8 & 2.2 & 4.8 & 11.1 & 23.1 & 13.3 \\
\hline $\mathrm{Ce}$ & 6 & 15 & 16 & 5 & 12 & 27 & 51.3 & 29.9 \\
\hline $\mathrm{Pr}$ & na & na & na & па & na & na & 5.88 & 3.58 \\
\hline Nd & 5 & 9 & 9 & 5 & 10 & 20 & 28.1 & 18.5 \\
\hline Sm & 1.4 & 3.46 & 2.43 & 1.1 & 2.6 & 5.03 & 7.2 & 5 \\
\hline Eu & 0.69 & 1.3 & 0.8 & 0.4 & 0.93 & 1.4 & 2.27 & 1.72 \\
\hline Gd & na & na & na & na & na & na & 7.7 & 5.6 \\
\hline $\mathrm{Tb}$ & 0.5 & 0.9 & 0.7 & 0.5 & 0.6 & 1.1 & 1.3 & 0.9 \\
\hline Dy & na & na & na & na & na & na & 8 & 5.8 \\
\hline Ho & ná & na & na & na & na & na & 1.6 & 1.2 \\
\hline $\mathrm{Er}$ & na & na & na & na & na & na & 4.5 & 3.4 \\
\hline $\operatorname{Tm}$ & na & na & na & na & na & na & 0.66 & 0.49 \\
\hline $\mathrm{Yb}$ & 1.4 & 2.9 & 2.5 & 1.14 & 2.15 & 3.8 & 4.1 & 2,9 \\
\hline Lu & 0.22 & 0.42 & 0.37 & 0.19 & 0.35 & 0.51 & 0.66 & 0.46 \\
\hline$(\mathrm{La} / \mathrm{Yb}) \mathrm{N}$ & 0.92 & 1.31 & 1.84 & 1.31 & 1.51 & 1.98 & 3.82 & 3.11 \\
\hline
\end{tabular}


Table 2 - Chemical composition of the Intermediate Massive Granulites. na - not analyzed; nd - not detected; (13) - estimated values

Tabela 2 - Composição Química dos Granulitos Intermediários Maciços, na - não analisado; nd - não detectado; (13) - valores estimados

\begin{tabular}{|c|c|c|c|c|c|c|c|c|c|}
\hline Sample & MB - 99D & MB - 15B & MB - 19 & $M B-32 A$ & $\mathrm{MB}$ - 32D & $\mathrm{MB} \cdot 145 \mathrm{~B}$ & $\mathrm{MB}-297 \mathrm{D}$ & MB - 297B & $\mathrm{MB}-115 \mathrm{~B}$ \\
\hline $\mathrm{SiO}_{2}$ & 54.99 & 57.95 & 61.12 & 61.80 & 62.82 & 63.79 & 64.35 & 64.44 & 66.24 \\
\hline $\mathrm{TiO}_{2}$ & 0.98 & 0.86 & 0.92 & 1.03 & 1.13 & 0.78 & 0.70 & 0.63 & 0.52 \\
\hline $\mathrm{Al}_{2} \mathrm{O}_{3}$ & 16.70 & 16.41 & 15.37 & 16.42 & 15.76 & 15.15 & 15.60 & 16.39 & 15.41 \\
\hline $\mathrm{FeO}^{*}$ & 7.89 & 6.74 & 5.67 & 6.14 & 6.19 & 6.24 & 6.25 & 5.15 & 4.21 \\
\hline Mno & 0.13 & 0.11 & 0.10 & 0.11 & 0.12 & 0.09 & 0.12 & 0.07 & 0.07 \\
\hline $\mathrm{MgO}$ & 3.74 & 3.22 & 2.71 & 1.93 & 1.94 & 2.78 & 2.50 & 2.09 & 2.50 \\
\hline $\mathrm{CaO}$ & 6.24 & 4.41 & 4.34 & 3.69 & 3.77 & 4.97 & 3.01 & 3.24 & 4.34 \\
\hline $\mathrm{Na}_{2} \mathrm{O}$ & 3.58 & 3.90 & 4.24 & 4.08 & 3.72 & 3.05 & 4,34 & 4.65 & 4.51 \\
\hline $\mathrm{K}_{2} \mathrm{O}$ & 1.91 & 2.32 & 1.90 & 3.10 & 3.31 & 1.66 & 2.20 & 2.00 & 1.19 \\
\hline $\mathrm{P}_{2} \mathrm{O}_{5}$ & 0.31 & 0.18 & 0.34 & 0.40 & 0.39 & 0.20 & 0.19 & 0.12 & 0.22 \\
\hline LOI & 0.20 & 0.79 & 0.26 & 0.21 & 0.30 & 0.50 & 0.51 & 0.32 & 0.30 \\
\hline Total & 96.47 & 96.10 & 96.71 & 98.70 & 99.15 & 98.90 & 99.26 & 98.78 & 99.98 \\
\hline Mg\# & 45.79 & 45.99 & 46.00 & 35.91 & 35.83 & 44.27 & 41.60 & 41.98 & 51.41 \\
\hline $\mathrm{Cr}$ & na & 95 & 79 & 23 & 17 & na & 113 & 122 & na \\
\hline $\mathrm{Ni}$ & 17 & 36 & 26 & 27 & 26 & 48 & 36 & 57 & 45 \\
\hline Co & na & 23 & 16 & 15 & na & na & 19 & 20 & na \\
\hline $\mathrm{Sc}_{\mathrm{c}}$ & 23 & 15 & 13 & 9 & $\mathrm{na}$ & 12 & 15 & 11 & 8 \\
\hline$v$ & 103 & 89 & 74 & 60 & 77 & 81 & 94 & 91 & 51 \\
\hline $\mathrm{Cu}$ & 47 & 30 & 13 & 45 & 45 & 100 & 18 & 13 & 76 \\
\hline $\mathbf{P b}$ & 22 & 13 & 16 & 19 & 20 & 21 & 27 & 17 & 15 \\
\hline $\mathbf{Z n}$ & 83 & 82 & 86 & 90 & 95 & 69 & 77 & 76 & 71 \\
\hline $\mathbf{K}$ & 15855 & 19259 & 15772 & 25734 & 27477 & 13780 & 18263 & 16603 & 9879 \\
\hline $\mathbf{R b}$ & 40 & 96 & 34 & 89 & 86 & 59 & 87 & 71 & 70 \\
\hline $\mathrm{Cs}$ & nd & 1.50 & nd & 0.50 & na & nd & 2.10 & 0.50 & 1.10 \\
\hline $\mathrm{Ba}$ & 990 & 448 & 800 & 1210 & 1263 & 492 & 740 & 890 & 723 \\
\hline Sr & 570 & 346 & 470 & 327 & 334 & 303 & 527 & 667 & 637 \\
\hline $\mathbf{T a}$ & nd & nd & nd & 1.40 & 1.62 & nd & 0.44 & 0.19 & nd \\
\hline $\mathrm{Nb}$ & na & na & na & 23.8 & 27.6 & na & 8.2 & 6.5 & na \\
\hline Hf & 6.90 & 4.50 & 7.40 & (8.30) & nd & 5.00 & 9.80 & 3.80 & 4.80 \\
\hline $\mathrm{Zr}$ & 201 & 223 & 285 & 349 & 335 & 193 & 315 & 142 & 220 \\
\hline $\mathrm{Ti}$ & 5875 & 5156 & 5515 & 6175 & 6774 & 4676 & 4197 & 3777 & 3117 \\
\hline$Y$ & 23 & 24 & 30 & 54 & 39 & 25 & 18 & 6 & 8 \\
\hline Th & nd & 11.00 & 14.00 & 4.90 & na & 1.40 & 7.94 & 6.39 & 5.70 \\
\hline $\mathrm{u}$ & nd & 1.30 & nd & 0.20 & na & nd & 1.52 & 0.46 & nd \\
\hline $\mathrm{La}$ & 34.30 & 81.50 & 74.50 & 64.30 & na & 23.70 & 35.30 & 35.70 & 52.70 \\
\hline $\mathrm{Ce}$ & 67.00 & 136.00 & 125,00 & 108.00 & na & 45.00 & 67.60 & 64.60 & 86.00 \\
\hline $\mathbf{P r}$ & na & na & na & na & na & na & 6.62 & 6.08 & na \\
\hline Nd & 33.00 & 48.00 & 51.00 & 44.00 & na & 23.00 & 26.70 & 23.90 & 33.00 \\
\hline $\mathrm{Sm}$ & 6.33 & 5.90 & 7.50 & 7.20 & na & 4.65 & 4.60 & 3.80 & 4.47 \\
\hline Eu & 1.50 & 1.49 & 1.86 & 1.78 & na & 1.20 & 1.17 & 1.42 & 1.00 \\
\hline $\mathrm{Gd}$ & na & na & na & na & na & na & 3.40 & 2.20 & na \\
\hline $\mathbf{T b}$ & 0.80 & 0.50 & 0.90 & 1.20 & na & 0.80 & 0.50 & 0.20 & na \\
\hline Dy & na & na & na & na & na & na & 3.00 & 1.10 & na \\
\hline Ho & na & na & na & na & na & na & 0.60 & 0.20 & na \\
\hline Er & na & na & na & na & na & na & 1.90 & 0.50 & na \\
\hline $\mathbf{T m}$ & na & na & na & na & na & na & 0.28 & 0.06 & na \\
\hline $\mathrm{Yb}$ & 2.10 & 0.96 & 1.32 & 3.83 & na & 2.40 & 1.80 & 0.40 & 0.60 \\
\hline Lu & 0.30 & 0.15 & 0.23 & 0.60 & na & 0.31 & 0.30 & 0.08 & 0.11 \\
\hline$(\mathrm{La} / \mathrm{Yb}) \mathrm{N}$ & 11.07 & 57.53 & 38.21 & 11.37 & $=$ & 6.69 & 13.29 & 60.61 & 59.44 \\
\hline
\end{tabular}


Table 3 - Chemical composition of the Intermediate Gneissic and Migmatitic Granulites. na - not analyzed; nd - not detected; (13) - estimated values

Tabela 3 - Composição química dos Granulitos Intermediários Gnáissicos e Migmatíticos. na - não analisado; nd - não detectado; (13) - valores estimados

\begin{tabular}{|c|c|c|c|c|}
\hline Sample & $M B-43 C$ & $\mathrm{MB}-46 \mathrm{C}$ & $\mathrm{MB}-\mathbf{4 6 A}$ & $M B-46 T$ \\
\hline $\mathrm{SiO}_{2}$ & 55.90 & 58.07 & 61.59 & 62.40 \\
\hline $\mathrm{TiO}_{2}$ & 1.43 & 0.66 & 1.13 & 0.38 \\
\hline $\mathrm{Al}_{2} \mathrm{O}_{3}$ & 16.42 & 17.65 & 14.51 & 16.25 \\
\hline FeO* & 9.71 & 5.69 & 6.73 & 5.64 \\
\hline $\mathrm{MnO}$ & 0.27 & 0.11 & 0.12 & 0.13 \\
\hline $\mathrm{MgO}$ & 3.55 & 3.76 & 4.37 & 3.01 \\
\hline $\mathrm{CaO}$ & 4.78 & 3.80 & 4.64 & 4.49 \\
\hline $\mathrm{Na}_{2} \mathrm{O}$ & 4.18 & 5.23 & 4.10 & 4.99 \\
\hline $\mathrm{K}_{2} \mathrm{O}$ & 1.95 & 2.24 & 0.76 & 1.02 \\
\hline $\mathrm{P}_{2} \mathrm{O}_{5}$ & 0.49 & 0.17 & 0.31 & 0.48 \\
\hline LOI & 0.74 & 0.78 & 0.08 & 0.40 \\
\hline Total & 100.50 & 98.79 & 99.09 & 99.82 \\
\hline $\mathrm{Mg} \#$ & 39.45 & 54.09 & 53.64 & 48.74 \\
\hline $\mathrm{Cr}$ & 163 & 242 & 130 & ла \\
\hline $\mathrm{Ni}$ & 111 & 79 & 92 & 68 \\
\hline $\mathrm{Sc}$ & na & na & na & 11 \\
\hline $\mathrm{V}$ & 132 & 105 & 171 & 66 \\
\hline $\mathrm{Cu}$ & 46 & 26 & 28 & 61 \\
\hline $\mathbf{P b}$ & 18 & 26 & 21 & 21 \\
\hline $\mathrm{Zn}$ & 120 & 97 & 97 & 78 \\
\hline $\mathbf{K}$ & 16188 & 18595 & 6309 & 8467 \\
\hline $\mathbf{R b}$ & 27 & 73 & 32 & 21 \\
\hline $\mathrm{Ba}$ & 983 & 525 & 523 & 220 \\
\hline $\mathbf{S r}$ & 465 & 360 & 198 & 303 \\
\hline $\mathrm{Ta}$ & 0.73 & 0.55 & 0.86 & 0.60 \\
\hline $\mathbf{N b}$ & 12.4 & 9.4 & 14.6 & $(10,2)$ \\
\hline Hf & na & na & na & 0.70 \\
\hline $\mathbf{Z r}_{\mathbf{r}}$ & 279 & 83 & 156 & 17 \\
\hline $\mathbf{T i}$ & 8573 & 3957 & 6774 & 2278 \\
\hline $\mathbf{Y}$ & 33 & 10 & 37 & 27 \\
\hline Th & na & na & na & 6.00 \\
\hline $\mathbf{U}$ & na & na & na & 1.60 \\
\hline La & na & na & na & 27.20 \\
\hline $\mathrm{Ce}$ & na & na & na & 53.00 \\
\hline Nd & $\mathbf{n a}$ & na & na & 28.00 \\
\hline $\mathrm{sm}$ & na & na & na & 6.23 \\
\hline Eu & na & na & na & 0.90 \\
\hline $\mathrm{Tb}$ & na & na & na & 0.90 \\
\hline $\mathrm{Yb}$ & na & na & na & 1.60 \\
\hline Lu & na & na & na & 0.22 \\
\hline$(\mathrm{L} a / \mathrm{Yb}) \mathrm{N}$ & - & - & - & 11.51 \\
\hline
\end{tabular}

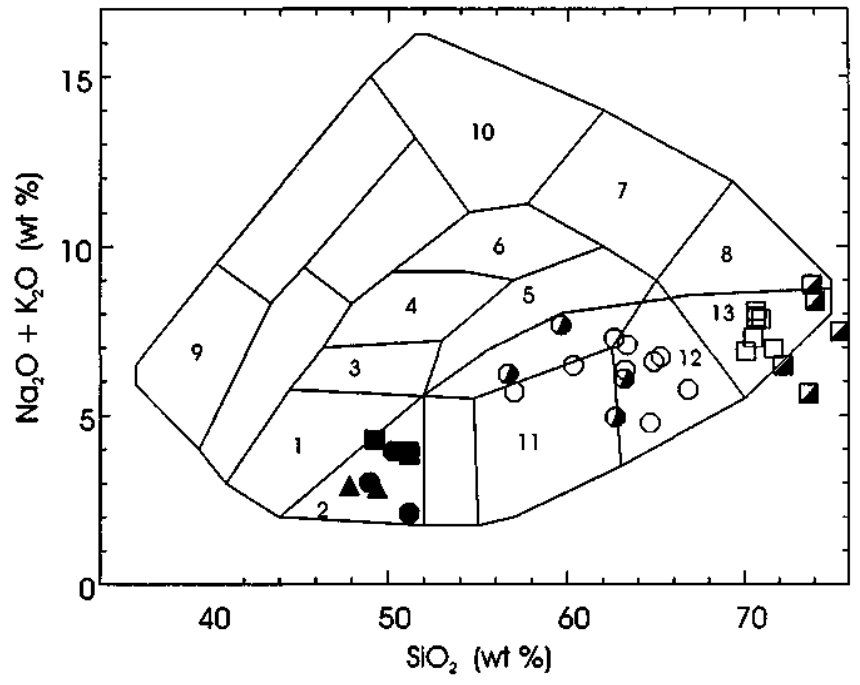

Figure 2 - Chemical classification of the rocks of the JFC (Cox et al. 1979); the dividing Une between the alkalic and subalkalic magma series is from Miyashiro (1978). 1,2, 3 Gabbro; 4,5 - Syeno-diorite; 6,7 - Syenite; 8 - Alkali-granite; 9 - Ijolite; 10 - Nepheline syenite; 11 - Diorite; 12 - Quartzdiorite (Granodiorite); 13 - Granite. Symbols: Mafic granulites (closed triangles, circles and squares); Intermediate granulites (half-closed circles - massive; and open circles

- gneissic and migmatitic granulites); Felsic granulites (open squares -charnockitic leucosomes and gneissic charnockites; and half - closed squares - intrusive charnockites).

Figura 2 - Classificação química das rochas do Complexo Juiz de Fora (CJF) (Cox et al. 1979); a linha divisória dos campos alcalino e subalcalino foi proposta por Miyashiro (1978). 1, 2, 3 - Gabro; 4, 5 - Sieno-diorito; 6, 7 Sienito; 8 - Álcali-granito; 9 - Ijolito; 10 - Nefelina sienito; 11 - Diorito; 12 - Quartzo-diorito (Granodiorito); 13 - Granito. Símbolos: Granulitos básicos (triângulos, círculos e quadrados fechados); Granulitos intermediários (círculos abertos - granulitos gnáissicos e migmatíticos; círculos parcialmente fechados - granulitos maciços); Granulitos ácidos (quadrados abertos - leucossomas charnockíticos e gnáissicos; quadrados parcialmente preenchidos - charnockitos intrusivos).

diorites, tonalites, and granodiorites, and that the felsic granulites are granodiorites and granites. A compositional gap occurs between 66,2 and $69,5 \% \mathrm{SiO}_{2}$ (see below), separating less from more silicic granodiorites. Trondhjemitic compositions were not found (Fig. 5), but studies on the possibility of the JFC being, to some extent, a granulitized TTG association are in course.

Figures 6 and 7 show that ali the studied granulites are subalkalic rocks, and that the mafic granulites delineate a tholeiitic trend, while the intermediate and felsic granulites have a calc-alkaline character. The main characteristics of each individual group are presented below.

MAFIC GRANULITES The mafic granulites have a basaltic composition (Fig. 2). Major and trace element abundance alio $\mathrm{w}$ the recognition of three distinct subgroups (Figs. 8, 9 and 10), which comprise (i) high-Mg tholeiites (HMgT), characterized by higher $\mathrm{MgO}$ and $\mathrm{CaO}$, and lower $\mathrm{FeO}, \mathrm{Sr}$, HFSE (and also REE; see below) contents as compared to the other tholeiites; (ii) a medium-Mg tholeiite (MMgT) with a composition which is intermediate between the $\mathrm{HMgT}$ and the low-Mg tholeiite (LMgT); and (iii) the LMgT, character- 
Table 4 - Chemical composition of the Charnockitic Leucosomes and Gneissic Charnockites. na - not analysed; nd - not detected; (13) - estimated values

Tabela 4 - Composição química dos Leucossomas Charnockíticos e Charnockitos gnáissicos. na - não analisado; nd - não detectado; (13) - valores estimados

\begin{tabular}{|c|c|c|c|c|c|c|c|}
\hline Sample & $\mathrm{MB}-\mathbf{3 2 C}$ & $\mathrm{MB}-241 \mathrm{~A}$ & MB - 48A & MB - 514E & $\mathrm{MB}$ - 151B & MB - 32B & MB - 514A \\
\hline $\mathrm{SiO}_{2}$ & 68.77 & 69.00 & 69.49 & 70.17 & 70.18 & 70.82 & 70.79 \\
\hline $\mathrm{TiO}_{2}$ & 0.43 & 0.35 & 0.55 & 0.40 & 0.51 & 0.36 & 0.34 \\
\hline $\mathrm{Al}_{2} \mathrm{O}_{3}$ & 14.29 & 14.90 & 14.76 & 15.22 & 14.80 & 15.06 & 15.08 \\
\hline $\mathrm{FeO}^{*}$ & 2.66 & 2.11 & 2.87 & 2.74 & 2.58 & 2.54 & 2.07 \\
\hline $\mathrm{MnO}$ & 0.05 & 0.03 & 0.06 & 0.03 & 0.04 & 0.05 & 0.03 \\
\hline $\mathrm{MgO}$ & 0.84 & 0.75 & 1.30 & 0.84 & 0.84 & 0.71 & 0.67 \\
\hline $\mathrm{CaO}$ & 2.46 & 2.37 & 3.05 & 2.71 & 2.39 & 2.46 & 2.81 \\
\hline $\mathrm{Na}_{2} \mathrm{O}$ & 3.17 & 3.82 & 4.39 & 3.76 & 3.70 & 3.38 & 4.00 \\
\hline $\mathrm{K}_{2} \mathrm{O}$ & 4,48 & 3.80 & 2.43 & 3.49 & 4.14 & 4.72 & 2.89 \\
\hline $\mathrm{P}_{2} \mathrm{O}_{5}$ & 0.12 & 0.12 & 0.24 & 0.17 & 0.16 & 0.16 & 0.11 \\
\hline LOI & 0.31 & 0.50 & 0.28 & 0.31 & 0.35 & 0.39 & 0.31 \\
\hline Total & 97.88 & 97.98 & 99.74 & 100.15 & 99.98 & 100.93 & 99.33 \\
\hline $\mathrm{Mg} \#$ & 35.98 & 38.83 & 44.66 & 35.29 & 36.69 & 33.27 & 36.58 \\
\hline $\mathrm{Cr}$ & 13 & na & 25 & 23 & na & 8 & 20 \\
\hline $\mathrm{Ni}$ & 11 & 3 & 14 & 9 & 2 & 9 & 19 \\
\hline Co & 8 & na & na & 6 & na & 5 & 6 \\
\hline Sc & 1 & 2 & na & 4 & 5 & 2 & 3 \\
\hline $\mathrm{v}$ & 21 & 28 & 46 & 30 & 21 & 17 & 50 \\
\hline $\mathrm{Cu}$ & 14 & 5 & 8 & 500 & 5 & 17 & 19 \\
\hline $\mathrm{Pb}$ & 24 & 26 & 23 & 74 & 23 & 22 & 26 \\
\hline $\mathrm{Zn}$ & 36 & 43 & 48 & 26 & 32 & 37 & 24 \\
\hline $\mathbf{K}$ & 37190 & 31545 & 20172 & 28972 & 34367 & 39182 & 23991 \\
\hline $\mathbf{R b}$ & 110 & 116 & 53 & 67 & 110 & 97 & 54 \\
\hline $\mathrm{Cs}$ & 0.50 & na & nd & na & nd & nd & nd \\
\hline $\mathrm{Ba}$ & 1896 & 1200 & 772 & 1644 & 1466 & 1478 & 1836 \\
\hline $\mathrm{Sr}$ & 303 & 402 & 358 & 652 & 415 & 290 & 520 \\
\hline $\mathrm{Tt}$ & na & na & na & 0.50 & na & na & 0.40 \\
\hline $\mathrm{Ga}$ & na & na & na & 19 & na & na & 19 \\
\hline $\mathrm{Ta}$ & nd & 0.50 & 0.39 & 0.21 & nd & 0.70 & 0.19 \\
\hline $\mathrm{Nb}$ & (8.5) & $(8.5)$ & $(6.6)$ & 5.5 & nd & 11.9 & 4.5 \\
\hline Hf & 5.20 & 6.70 & nd & 5.50 & 4.70 & 5.80 & 4.40 \\
\hline $\mathrm{Zr}$ & 226 & 181 & 208 & 222 & 262 & 230 & 174 \\
\hline $\mathrm{Ti}$ & 2578 & 2098 & 3297 & 2398 & 3057 & 2158 & 2038 \\
\hline$Y$ & 10 & 8 & 10 & 7 & 11 & 22 & 5 \\
\hline Th & 0.50 & 25.70 & nd & 25.77 & 15.70 & 7.30 & 1.04 \\
\hline $\mathrm{U}$ & 0.60 & 0.90 & na & 0.47 & nd & 0.70 & 0.39 \\
\hline $\mathbf{L a}$ & 24.20 & 77.90 & na & 101.20 & 49.90 & 49.10 & 23.90 \\
\hline $\mathrm{Ce}$ & 35.00 & 117.00 & na & 165.60 & 73.00 & 79.00 & 35.60 \\
\hline Pr & na & na & na & 14.19 & na & na & 3.04 \\
\hline Nd & 12.00 & 41.00 & na & 50.20 & 25.00 & 30.00 & 10.80 \\
\hline Sm & 1.90 & 5.59 & na & 6.50 & 3.69 & 4.10 & 1.90 \\
\hline Eu & 1.25 & 1.30 & na & 1.36 & 1.10 & 1.38 & 1.12 \\
\hline Gd & na & na & na & 3.20 & na & na & 1.40 \\
\hline $\mathrm{Tb}$ & 0.50 & nd & na & 0.30 & 0.60 & 0.50 & 0.20 \\
\hline Dy & na & na & na & 1.60 & na & na & 0.80 \\
\hline Ho & na & na & na & 0.30 & na & na & 0.20 \\
\hline $\mathrm{Er}$ & na & na & na & 0.50 & na & na & 0.40 \\
\hline Tm & na & na & na & 0.06 & na & na & nd \\
\hline $\mathrm{Yb}$ & 1.21 & 0.70 & na & 0.30 & 0.50 & 1.08 & 0.40 \\
\hline Lu & 0.22 & 0.12 & na & 0.08 & 0.08 & 0.20 & 0.06 \\
\hline$(\mathrm{L} a / \mathrm{Yb}) \mathrm{N}$ & 13.55 & 75.46 & - & 228.29 & 67.63 & 30.82 & 40.58 \\
\hline
\end{tabular}


Table 5 - Chemical composition of the Intrusive Charnockites. na - not analyzed; nd - not detected; (13) - estimated values Tabela 5 - Composição Química dos Charnockitos Intrusivos. na - não analisado; nd - não detectado.

\begin{tabular}{|c|c|c|c|c|c|c|c|}
\hline Sample & MB - 151 A & $\mathrm{MB}$ - 43B & MB - 37 (1) & $M B-46 R$ & $\mathrm{MB}-\mathbf{4 6 B}$ & $\mathrm{MB}-46 \mathrm{U}$ & $M B-43 A$ \\
\hline $\mathrm{SiO}_{2}$ & 72.05 & 73.61 & 71.50 & 71.92 & 74.55 & 74.97 & 79.43 \\
\hline $\mathrm{TiO}_{2}$ & 0.21 & 0.19 & 0.53 & 0.19 & 0.08 & 0.02 & 0.19 \\
\hline $\mathrm{Al}_{2} \mathrm{O}_{3}$ & 13.75 & 13.59 & 14.02 & 13.98 & 13.66 & 13.81 & 11.63 \\
\hline $\mathrm{FeO}^{*}$ & 1.23 & 1.30 & 2.65 & 1.88 & 0.74 & 0.25 & 1.23 \\
\hline $\mathrm{MnO}$ & 0.02 & 0.02 & 0.04 & 0.03 & 0.02 & nd & 0.02 \\
\hline $\mathrm{MgO}$ & 0.35 & 0.76 & 0.77 & 0.88 & 0.39 & 0.09 & 0.61 \\
\hline $\mathrm{CaO}$ & 1.47 & 1.30 & 3.00 & 3.18 & 1.92 & 1.98 & 3.05 \\
\hline $\mathrm{Na}_{2} \mathrm{O}$ & 3.30 & 2.56 & 3.22 & 4.22 & 3.70 & 3.71 & 3.22 \\
\hline $\mathrm{K}_{2} \mathrm{O}$ & 4.85 & 6.26 & 3.18 & 1.28 & 3.68 & 3.44 & 0.72 \\
\hline $\mathrm{P}_{2} \mathrm{O}_{5}$ & 0.05 & 0.04 & 0.15 & 0.07 & 0.03 & nd & 0.02 \\
\hline LOI & 0.20 & 0.32 & nd & 1.15 & 1.19 & 0.35 & 0.62 \\
\hline Total & 97.62 & 100.10 & 99.35 & 98.99 & 100.04 & 98.65 & 100.88 \\
\hline Mg \# & 33.60 & 50.93 & 34.15 & 45.47 & 48.50 & 38.90 & 46.86 \\
\hline $\mathrm{Cr}_{\mathbf{r}}$ & na & 39 & na & na & 9 & na & 13 \\
\hline $\mathrm{Ni}$ & 2 & 6 & 4 & 12 & 8 & 2 & 7 \\
\hline Sc & 1 & & 3 & 2 & na & nd & na \\
\hline$v$ & 10 & 28 & 23 & 24 & 11 & 2 & 25 \\
\hline $\mathrm{Cu}$ & 4 & 5 & 16 & 64 & 23 & 9 & 9 \\
\hline $\mathbf{P b}$ & 184 & 41 & 17 & 19 & 28 & 27 & 10 \\
\hline $\mathrm{Zn}$ & 17 & 22 & 35 & 19 & 9 & 4 & 11 \\
\hline K & 40261 & 51966 & 26398 & 10626 & 30549 & 28556 & 5977 \\
\hline $\mathbf{R b}$ & 145 & 94 & 80 & 24 & 79 & 75 & 5 \\
\hline $\mathrm{Ba}$ & 1543 & 1440 & 1486 & 385 & 1762 & 1659 & 373 \\
\hline $\mathrm{Sr}$ & 344 & 268 & 311 & 291 & 312 & 281 & 272 \\
\hline $\mathrm{Ta}$ & nd & 0.08 & nd & nd & 0.12 & nd & 0.11 \\
\hline $\mathrm{Nb}$ & nd & 1.4 & na & na & 2.0 & na & 1.8 \\
\hline $\mathrm{Hf}$ & 3.70 & nd & 6.20 & 3.40 & na & 2.00 & na \\
\hline $\mathrm{Zr}$ & 130 & 105 & 259 & 134 & 87 & 60 & 47 \\
\hline $\mathrm{Ti}$ & 1259 & 1139 & 3177 & 1139 & 480 & 120 & 1139 \\
\hline $\mathrm{Y}$ & 6 & 3 & 10 & 3 & 4 & nd & 2 \\
\hline Th & 21.30 & na & 2.50 & 0.40 & na & 3.50 & na \\
\hline $\mathbf{U}$ & nd & na & nd & nd & na & 1.00 & na \\
\hline $\mathbf{L a}$ & 42.30 & na & 32.00 & 14.50 & na & 13.30 & na \\
\hline $\mathrm{Ce}$ & 61.00 & na & 44.00 & 21.00 & na & 16.00 & na \\
\hline Nd & 20.00 & na & 16.00 & 10.00 & na & 8.00 & na \\
\hline Sm & 3.21 & na & 2.63 & 1.16 & na & 0.52 & na \\
\hline Eu & 0.80 & na & 1.40 & 0.80 & na & 0.80 & na \\
\hline $\mathrm{Yb}$ & 0.40 & na & 1.20 & 0.30 & na & nd & na \\
\hline Lu & 0.08 & na & 0.18 & 0.05 & na & nd & na \\
\hline$(\mathrm{La} / \mathrm{Yb}) \mathrm{N}$ & 71.82 & - & 18.08 & 30.61 & - & $>30.61$ & - \\
\hline
\end{tabular}




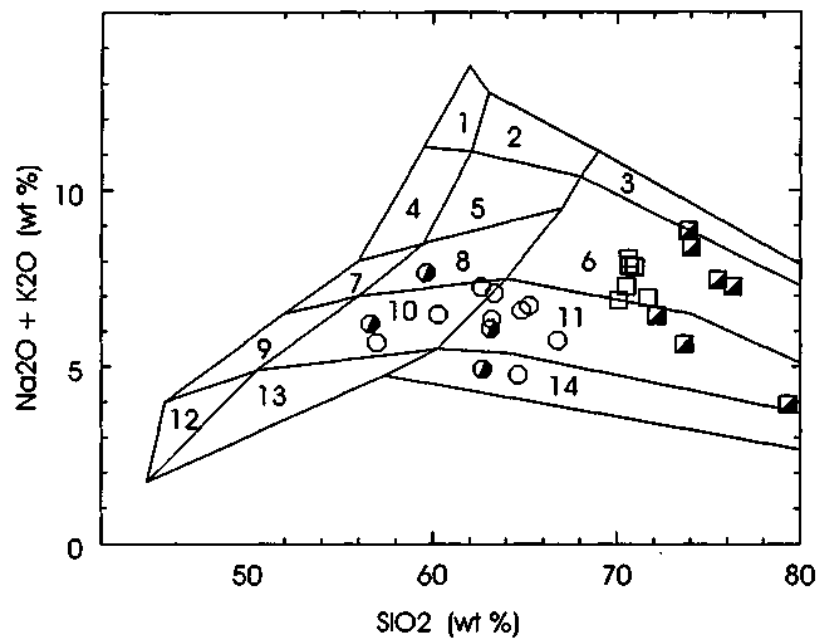

Figure 3 - Chemical classification of the rocks of the intermediate and felsic granulites (Middlemost 1985). 1 - Alkali-feldspar syenite; 2 - Alkali-feldspar quartz syenite; 3 - Alkalifeldspar granite; 4 - Syenite; 5 - Quartz syenite; 6 - Granite; 7 - Monzonite; 8 - Quartz monzonite; 9 - Monzodiorite; 10 Quartz monzonite; 11 -Granodioriíe; 12 - Diorite and gabbro; 13 - Quartz diorite; 14 - Tonalite. Symbols as in figure 2 .

Figura 3 - Classificação química dos granulitos intermediários e ácidos (Middlemost 1985). 1 -Álcai-feldspato sienito; 2 - Álcali-feldspato quartzo sienito; 3 - Álcali-feldspato granito; 4 - Sienito; 5 -Quartzo sienito; 6 Granito; 7 - Monzonito; 8 - Quartzo monzonito; 9 - Monzodiorito; 10 Quartzo monzonito; 11 -Granodiorito; 12 - Diorito e gabro; 13 - Quartzo diorito; 14 - Tonalito. Símbolos como na figura 2.

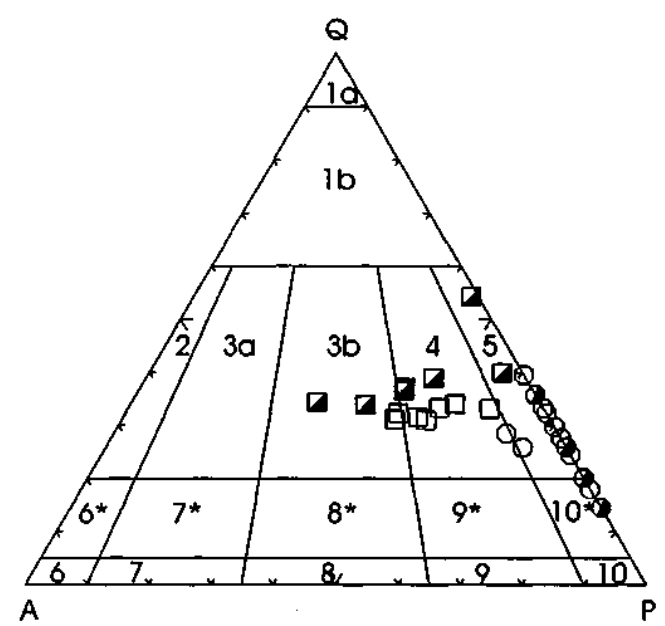

Figure 4 - Plots of the intermediate andfelsic granulites in the QAP normative diagram (Le Maitre 1989). $1 a$ and $1 b$ quartz-rich granitoids ; 2-Alkali feldspar granite; $3 a-$ Syenogranite; 3b-Monzogranite; 4-Granodiorite; 5Tonalite; 6-Alkali feldspar syenite; $6 *$-Nepeline-alkali feldspar syenite; 7-Syenite; 7*-Nepheline syenite; 8-Monzonite ; $8 *$-Latite; 9-Monzogabbro ; $9 *$ - Nepheline monzogabbro; 10-Diorite, anorthosite, gabbro, norite; $10^{*}$-Nepheline diorite, nepheline gabbro. Symbols as in figure 2. Figura 4 - Granulitos intermediários e ácidos em diagrama QAP normativo (Le Maitre 1989). 1a and 1b-granitóides ricos em quartzo; 2-Á lcali feldspato granito; 3a-Sienogranito; 3b-Monzogranito; 4-Granodiorito; 5-Tonalito; 6Álcali feldspato sienito; $6^{*}$-Nefelina-álcali feldspato sienito; 7-Sienito; $7 *$ Nefelina sienito; 8-Monzonito; 8*-Latito; 9-Monzogabro; 9*-Nefelina monzogabbro; 10-Diorito, anortosito, gabro, norito; $10 *$-Nefelina diorito, nefelina gabro. Símbolos como na figura 2.
15

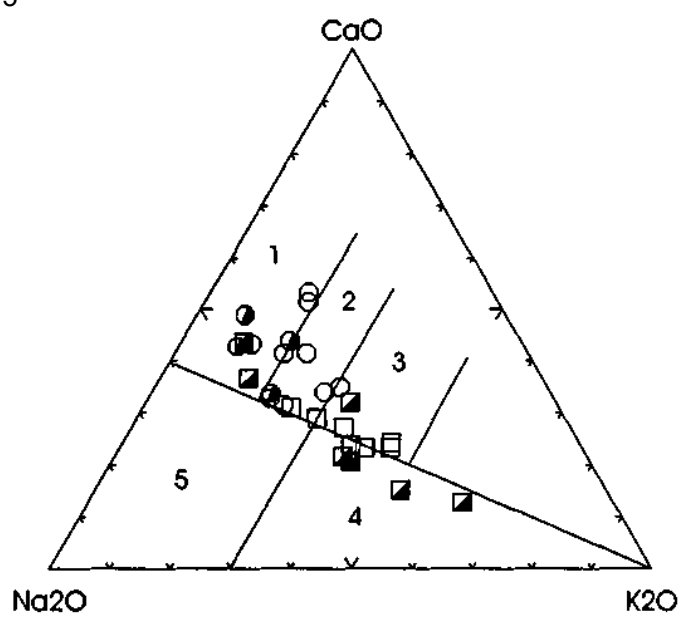

Figure 5 - Chemical classification of the intermediate and felsic granulites in $\mathrm{Na}_{2} \mathrm{O} \times \mathrm{CaO} \times \mathrm{K}_{2} \mathrm{O}$ (Glikson 1979). 1 Tonalite; 2 - Granodiorite; 3 - Adamellite; 4 - Granite; 5 Trondhjemite. Symbols as in figure 2.

Figura 5 - Classificação química dos granulitos intermediários e félsicos em diagrama $\mathrm{Na}_{2} \mathrm{O} \times \mathrm{CaO} \times \mathrm{K}_{2} \mathrm{O}$ (Glikson 1979). 1 - Tonalito; 2 - Granodiorito; 3 -Adamellito; 4 - Granito; 5 - Trondhjemito. Símbolos como na figura 2.

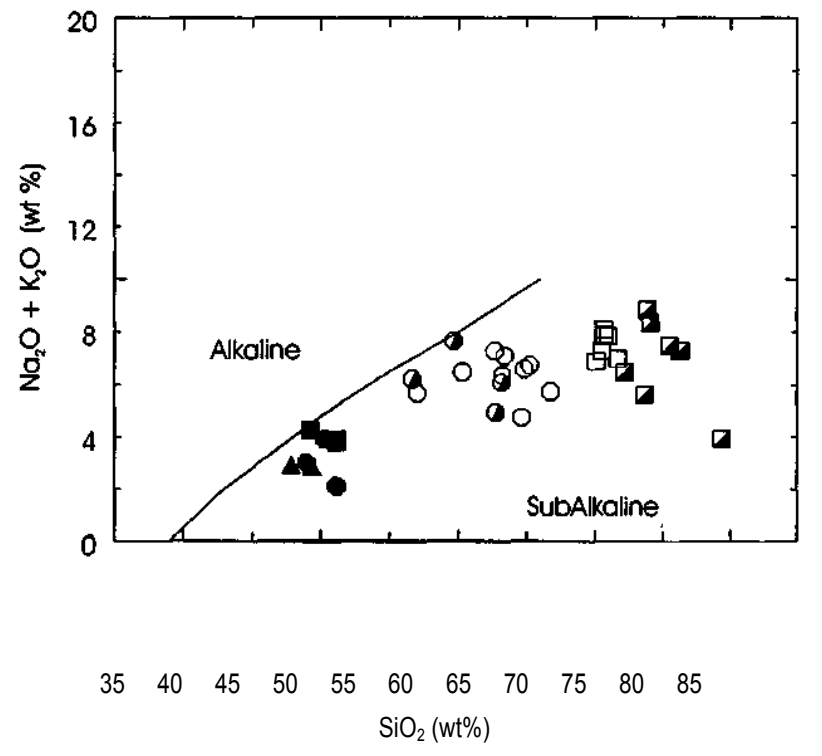

Figure $6-\mathrm{SiO}_{2} x \mathrm{Na}_{2} \mathrm{O}+\mathrm{K}_{2} \mathrm{O}$ diagram (Le Maitre 1989) with the dividing Une of the Alkaline and Subalkaline fields, proposed by Irvine \& Baragar (1971). Symbols as in figure 2. Figura 6 - Diagrama $\mathrm{SiO}_{2} \times \mathrm{Na}_{2} \mathrm{O}+\mathrm{K}_{2} \mathrm{O}$ (Le Maitre 1989), com a curva limite dos campos Alcalino e Subalcalino proposta por Irvine \& Baragar (1971). Símbolos como na figura 2.

ized by higher FeO, LILE, HFSE (and also the REE, see belo w). The HMgT occurs as centimetric enclaves in the intrusive charnockites, whereas the MMgT and the $\mathrm{LMgT}$ occur either as small bodies and as metric bands within ali the calc-alkaline rocks.

INTERMEDIATE AND FELSIC GRANULITES In the Harker diagrams (Fig. 9), the compositional gap between the intermediate and the felsic granulites is also evident when other oxides than $\mathrm{SiO}_{2}$ are considered. The behaviour of the more immobile elements ( $\mathrm{Mg}, \mathrm{Ti}, \mathrm{Ni}, \mathrm{V}$ and $\mathrm{Zr}$ ) suggest that 


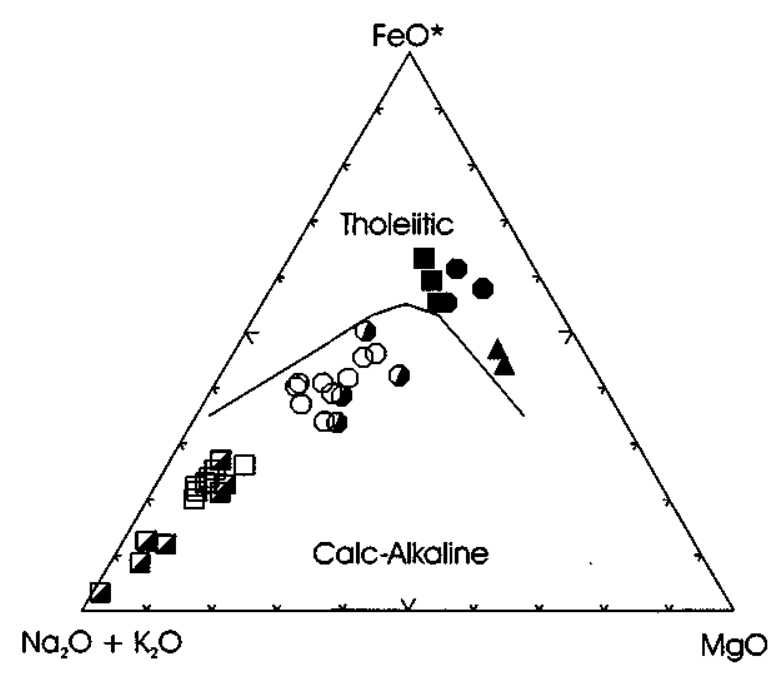

Figure 7 - AFM diagram (Irvine \& Baragar 1971). Symbols as in fgure 2.

Figura 7 - Diagrama AFM (Irvine \& Baragar 1971). Símbolos como na figura 2 .

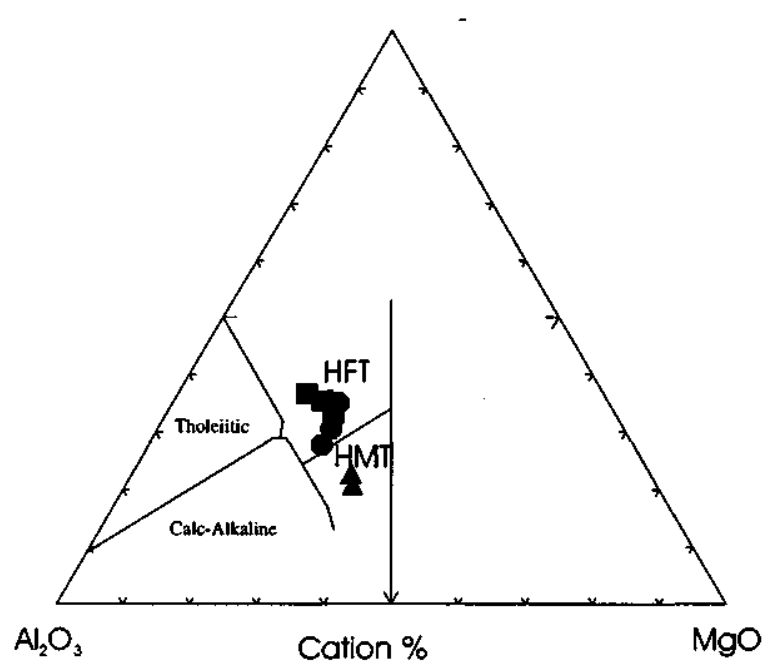

$\mathrm{FeO}^{*}+\mathrm{TiO}$

Figure 8 - Plot of the tholeiitic rocks in the cationic ternary diagram of Jensen (1976). HFT-high-Fe tholeiites; HMT high-Mg tholeiites. Symbols: High-Mg tholeiites (closed triangles); Medium-Mg tholeiites (closed circles); Low-Mg tholeiites (closed squares).

Figura 8 - Classificação das rochas toleíiticas em diagrama ternário catiônico proposto por Jensen (1976). Símbolos: Toleiitos de alto Mg (triângulos cheios); Toleítos de médio Mg (círculos cheios); Toleítos de baixo $\mathrm{Mg}$ (quadrados cheios).

each group has a characteristic magmatic trend. As compared to these elements, $\mathrm{Fe}, \mathrm{Ca}$, and $\mathrm{Al}$ also behaved as immobile elements, as suggested by the small or lack of scatter of these elements for each group of granulite. Some of the diagrams $(\mathrm{MgO}, \mathrm{FeO}$, $\mathrm{Ni}, \mathrm{V}$ and $\mathrm{Zr}$ ) suggest that the massive intermediate granulites are geochemically different from the gneissic and migmatitic intermediate ones. The former have higher contents of compatible elements ( $\mathrm{FeO}, \mathrm{MgO}$ and $\mathrm{Ni}$ ), $\mathrm{Na}_{2} \mathrm{O}$, and $\mathrm{V}$, and lower abundance of $\mathrm{Zr}$. Considering the more incompatible elements ( $\mathrm{K}, \mathrm{Rb}, \mathrm{Ba}, \mathrm{Sr})$, the massive granulites are more depleted than the other intermediate granulites. Scattering occurs in $\mathrm{Ba}, \mathrm{Rb}, \mathrm{Hf}$ and $\mathrm{Y}$.

A comparison between the two groups of felsic granulites shows that the charnockitic leucosomes and gneissic granulites have a very restrict $\mathrm{SiO}_{2}$ range, which is more variable in the intrusive charnockites. Additionally, the latter are richer in $\mathrm{MgO}, \mathrm{FeO}^{*}$, $\mathrm{Ni}$ and $\mathrm{V}$, poorer in $\mathrm{Sr}$ and $\mathrm{Y}$, and their $\mathrm{K}_{2} \mathrm{O}$. $\mathrm{Rb}$, and $\mathrm{Ba}$ variation is scattered.

REE Geochemistry The REE contents of the studied granulites are in Tables 1 to 5, and their patterns in figure 11.

MAFIC GRANULITES The chondrite-normalized REE patterns of the HMgT are roughly flat, 6 to 8 times chondrite. One of the patterns is slightly light-REE depleted (Fig. 1 Ia). In contrast, the REE patterns of the MMgT and the LMgT groups are, respectively, slightly and moderately fractionated, with absent to negative Eu anomalies. The LREEenriched rocks have a $[\mathrm{La} / \mathrm{Yb}] \mathrm{N}$ ratio (Table 1) that increases with decreasing $\mathrm{MgO}$. As this negative correlation is expected to take place during magmatic differentiation, the mafic granulites may have formed by differentiation from a single parental magma. However, considering that sample $17 \mathrm{~A}$ is slightly depleted in light-REE and that both 17A and $17 \mathrm{~B}$ are samples from the same outcrop, it is convenient to treat them as a separate group.

INTERMEDIATE GRANULITES Two distinct REE patterns (Fig. $11 \mathrm{~b}$ ) occur in the intermediate granulites. One is characterized by a moderate fractionation $([\mathrm{La} / \mathrm{Yb}] \mathrm{N}=6$ to 12 ), with slight to strong negative Eu anomalies (samples 99D, 32A, 46T and 145B), while the other has a strong fractionated pattern ([La/Yb]N $\approx 13$ to 60$)$, and lacks Eu anomaly. The lack of correlation between the degree of REE fractionation and $\mathrm{SiO}_{2}$ suggests that these rocks are not all genetically related.

FELSIC GRANULITES Charnockitic leucosomes and Gneissic Charnockites These granulites show two different REE patterns (Fig. $11 \mathrm{c}$ ). Two samples are moderatly fractionated $([\mathrm{La} / \mathrm{Yb}] \mathrm{N} \approx 13$ to 30$)$, with the most fractionated having a slight positive Eu anomaly, while the other lacks $\mathrm{Eu}$ anomaly. The remaining samples are more fractionated $([\mathrm{La} / \mathrm{Yb}] \mathrm{N} \approx 40$ to 230$)$, and the least fractionated sample hás a positive Eu anomaly, while the others lack Eu anomalies. Since this group of granulites hás a narrow $\mathrm{SiO}_{2}$ variation, $\mathrm{Na}_{2} \mathrm{O}$ was taken as an alternative differentiation index. The lack of correlation between the REE fractionation and $\mathrm{Na}_{2} \mathrm{O}$ suggests either that at least some of these rocks are not genetically related, or that $\mathrm{Na}_{2} \mathrm{O}$ is not an adequate differentiation index. REE patterns of samples 151B, $241 \mathrm{~A}, 514 \mathrm{E}$ and, to a lesser extent, 514A, resemble those of the highly fractionated intermediate granulites and, thus, could be genetically related.

Intrusive Charnockites Two distinct REE patterns are shown by these rocks (Fig. $11 \mathrm{~d}$ ). Most samples are moderatly fractionated $([\mathrm{La} / \mathrm{Yb}] \mathrm{N} 18->30)$, and have a positive $\mathrm{Eu}$ anomal that increses with the ammount of $\mathrm{SiO}_{2}$. Sample $46 \mathrm{U}$ displays a negative Sm anomaly, which is probably due to the fractionation of garnet. One sample (151 A) is highly fractionated, and lacks Eu anomaly, resembling the highly 


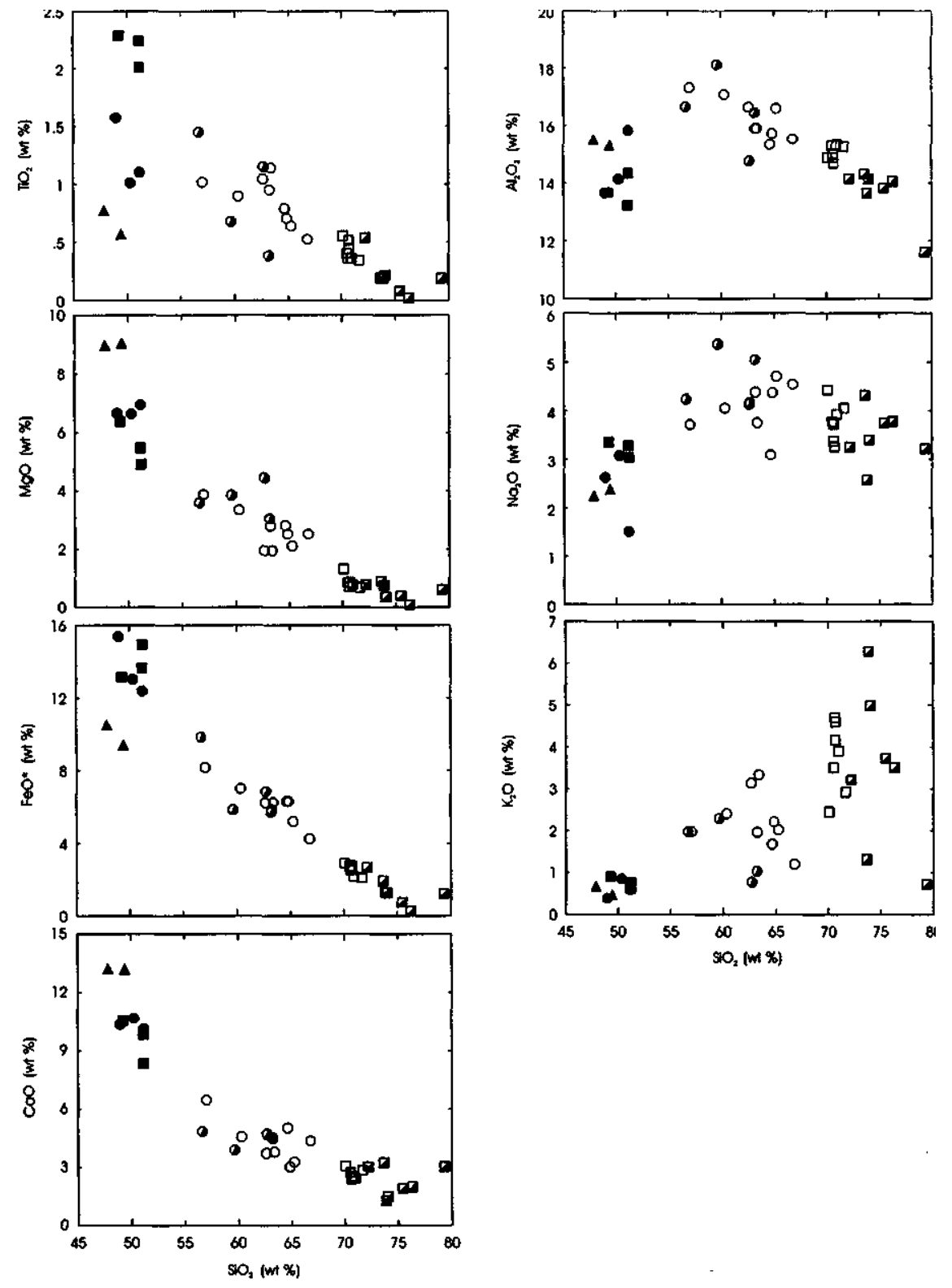

Figure 9 - Harker-type variation diagrams of the rocks from the JFC (major elements). Symbols as in figures 2 and 8.

Figura 9 - Diagramas de variação tipo Harker para as rochas do CJF (elementos maiores). Símbolos como nas figuras 2 e 8 .
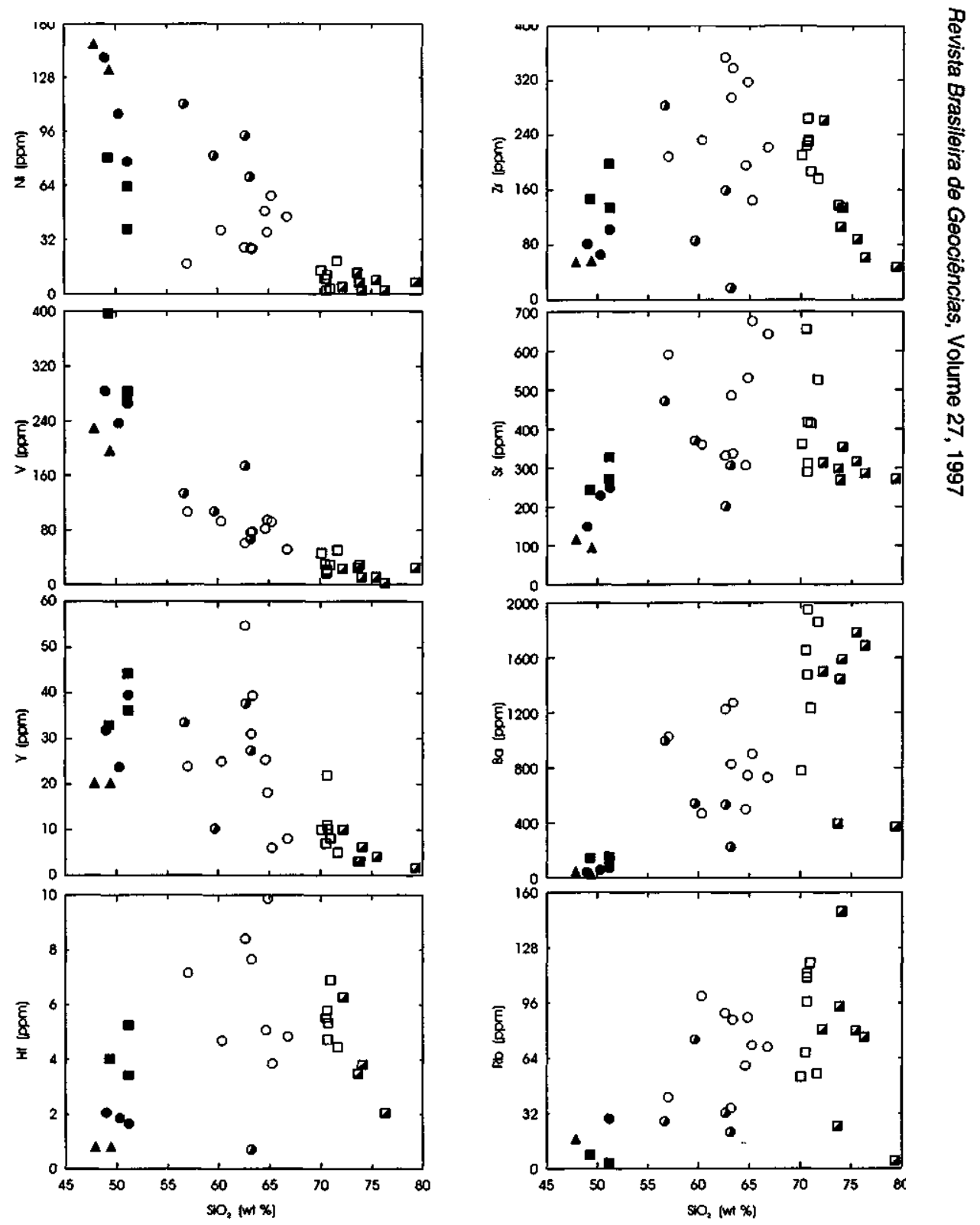

Figure 10 - Harker-type variation diagrams of the rocks from the JFC (trace elements). Symbols as in figures 2 and 8.

Figura 10 - Diagramas de variação tipo Harker para as rochas do CJF (elementos traços). Símbolos como nas figuras 2 e 8 . 

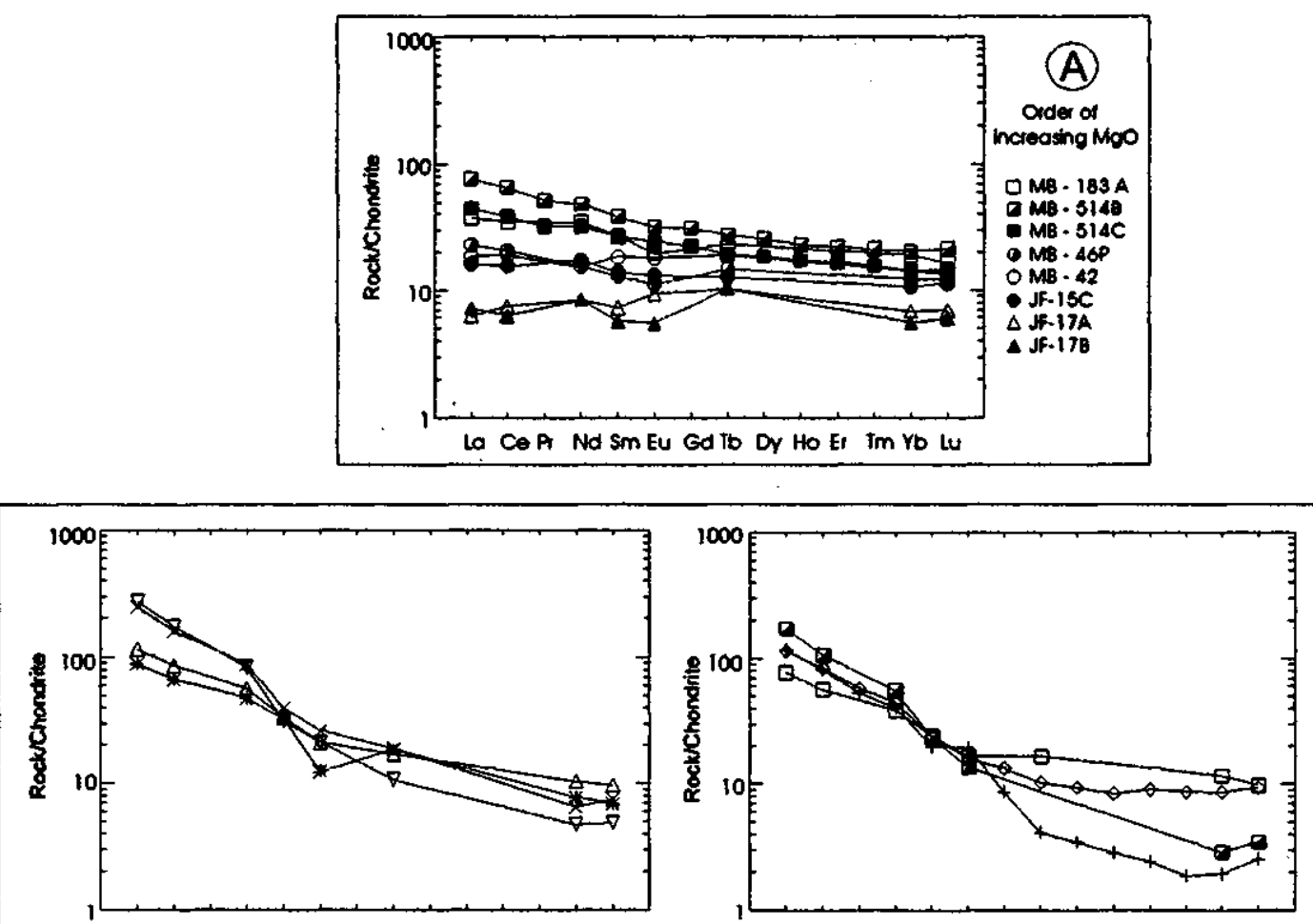

(B)

Order of decreang $\mathrm{SO}_{2}$

C MB - 1158

$+M B \cdot 2978$

- MB - 2970

प $M A \cdot 145 \mathrm{~B}$

C $\mathrm{NA} \cdot 19$

W. $M B$ - $46 \mathrm{~T}$

O. JF - 32A

JF - 158

$\triangle \mathrm{MB} \cdot 990$

Lo Co A Na Sin EU Gato Dy Ho E Tm Yo

ta CeP Na sm Eu Golo Dy Ho er Im ro
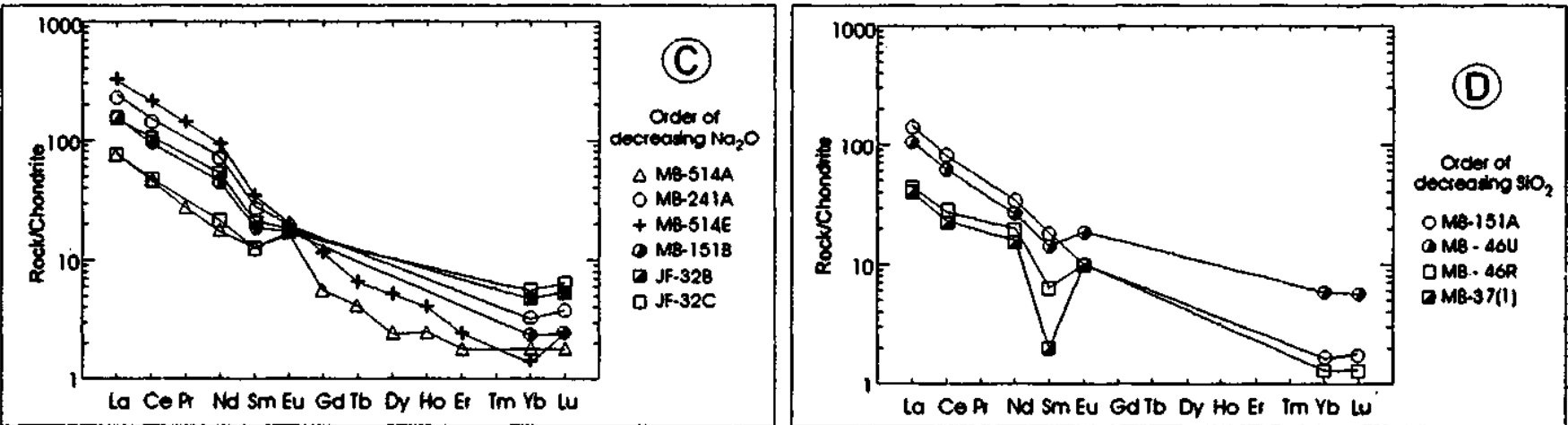

Figure 11 - Chondrite-normalized REE diagrams of the studied JFC granulites. Normalization to values of Boynton (1984). (a) Mafic granulites. Symbols: HMgT (triangles); MMgT (circles); LMgT (squares).(b) -Intermediate granulites. (c) - Charnokitic leucossomes and gneissic charnolites. (d) - Intrusive chanokites.

Figura 11 - Diagramas de ETR normalizados ao condrito dos granulitos do CJF. Normalização aos valores de Boyton (1984). (a) - Granulitos máficos. Símbolos: toleiitos de alto $\mathrm{Mg}$ (triângulos); toleiitos de médio $\mathrm{Mg}$ (círculos); toleiitos de baixo Mg (quadrados), (b) - Granulitos Intermediários, (c) - Leucossomas charnokíticos e gnáissicos. (d) Charnockitos intrusivos.

fractionated charnockitic leucosomes and gneissic charnockites, to which it might be cogenetic.

Tectonic Setting MAFIC GRANULITES NMORB normalized spidergrams (Fig. 12) indicate that the three groups of tholeiites are characterized by a selective enrichment in low ionic potential incompatible elements $(\mathrm{Sr}$, $\mathrm{K}, \mathrm{Rb}, \mathrm{Ba}, \mathrm{Th})$.

Considering most of the elements of high ionic potential (Ce, P, Zr, Hf, Sm, Ti, Y, Yb), the MMgT group shows N-MORB abundance, while the HMgT are strongly depleted. The Ta contents of the MMgT are similar to intra-plate tholeiites. The patterns of the above mentioned elements in the
HMgT resemble those of modern island are tholeiites, whereas the MMgT is similar to E-MORB. For comparison, typical island are and E-MORB patterns are included in the diagrams of $\mathrm{HMgT}$ and MMgT, respectively (Fig. 12).

In the N-MORB normalized variation diagram (Fig. 12), the LMgT has a hump-shaped pattern, characteristic of intraplate tholeiites. Plots of the Deccan flood basalts are shown to enable comparison.

The plots of the HMgT in tectonic discriminam diagrams (Fig. 13) are generally coincident with those of the low-K island are tholeiites.

In the diagrams of figures $13 \mathrm{a}, \mathrm{b}$ and $\mathrm{c}$, the MMgT plots in the field of ocean floor basalts. The trace element and REE 

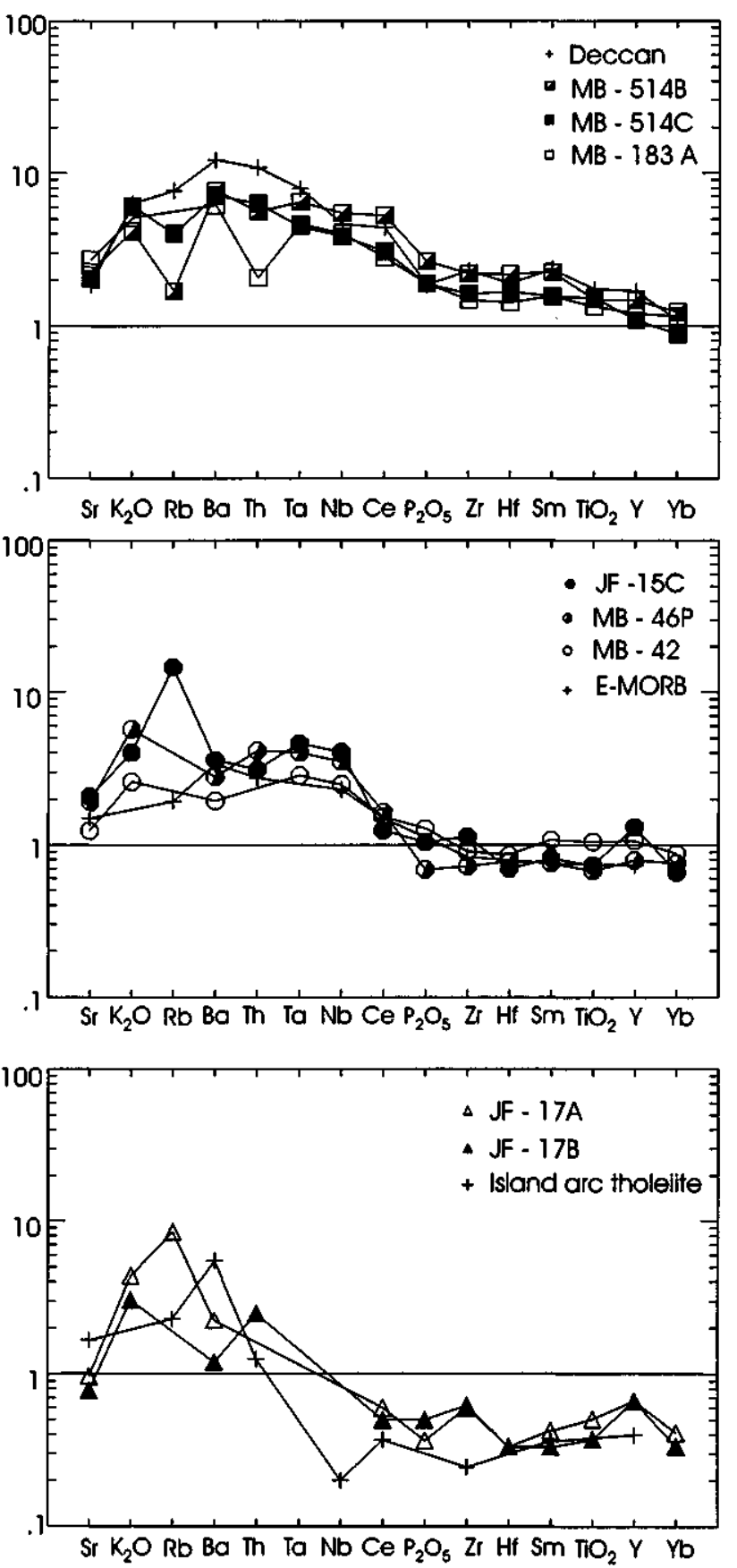

Figure 12 - MORB-normalized multi-element variation diagrams of the tholeiitic rocks (Pearce 1983). Symbols as in figure 11. Distribution patterns of the Deccan flood basalts (Thompson et al. 1983) and average modern E-MORB and islandarc tholeiites (Sun 1980) are shown for comparison. Figura 12 - Diagramas multi-elementares das rochas tholeiíticas, normalizados pelo MORB de Pearce 1983. Símbolos como na figura 11. Padrõ es de distribuição dos basaltos de platô do Deccan (Tompson et al. 1983) e de composições médias de E-MORB e arcos de ilhas modernos (Sun 1980) são mostrados para comparação.
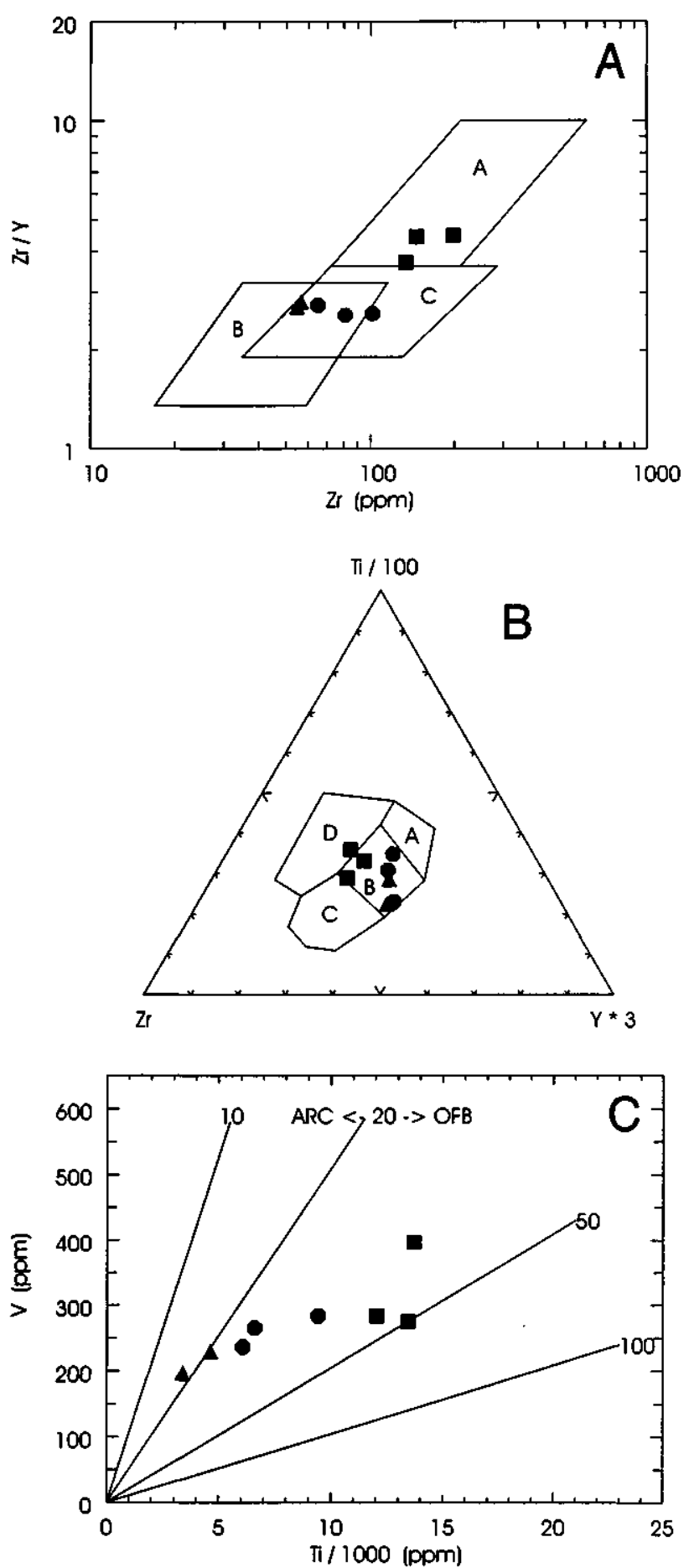

Figura 13 - Tectonic discriminant diagrams for the tholeiitic rocks. Symbols as in figures 2 and 8.(a) after Pearce \& Norry (1979). A - Within-plate basalts; $B$ - Island are basalts; $C$ Mid ocean ridge basalts. (b) - after Pearce and Cann (1973). A, $B$ - Low-K tholeiites; $B$ - Ocean floor basalts; $B, C$ Calcalkaline basalts; $D$ - Within-plate basalts. (c) - after Shervais (1982). ARC - Islandarc basalts; OFB - Ocean floor basalts.

Figura 13 -.Diagramas discriminantes de ambientes tectônicos para as rochas toleiíticas. Símbolos como nas figuras 2 e 8 . (a) - segundo Pearce \& Norry (1979). A - Basaltos intra-placa; B - Basaltos de arcos de ilha; C -Basaltos de cadeias meso-oceânicas. (b) - segundo Pearce \& Cann (1973). A, B Toleiitos de baixo K; B - Basaltos de fundo oceânico; B, C - Basaltos calcioalcalinos; D -Basaltos intra-placa. (c) - segundo Shervais (1982). ARC -basaltos de arcos de ilha; OFB - Basaltos de fundo oceânico. 
abundance of this group is similar to E-MORB rather than $\mathrm{N}-\mathrm{MORB}$. E-MORB signatures may be found in several extensional tectonic environments, such as mid-ocean ridge, back-arc and intra-plate continental settings.

The LMgT has within-plate characteristics and is similar to the Deccan flood basalts, leading to their interpretation as tholeiites of intra-continental plate regime.

Based on these and REE data, the MMgT and the LMgT are interpreted as derived from magmatic rocks of extensional tectonic settings, and probably represent distinct stages of magmatic differentiation. Moreover, considering that the $\mathrm{MMgT}$ and the LMgT occur as metric bands resembling dike and sill-like bodies, or as enclaves, within the calc-alkaline rocks of the study area, both groups are interpreted as withinplate tholeiitic basalts.

INTERMEDIATE AND FELSIC GRANULITES The intermediate granulites and the charnockitic leucosomes and gneissic charnockites plotted in Ocean Ridge Granite-normalized spidergram (Fig. 14) display, respectively, volcanic are and syn-collisional characteristics. The latter tectonic environment was expected for the charnockitic leucosomes, corroborating the interpretation, based on field data, that these rocks are anatectic products of continental rocks.

The intrusive charnockites have patterns that are similar, but somewhat depleted, to those of modern volcanic are and collisional granites. Thus, the JFC intermediate and felsic granulites are considered as magmatic products of convergent tectonic settings, representing different stages and/or different calc-alkaline island or cordilleran ares of pre-1.8 Ga. ages.

CONCLUSIONS The JFC orthogranulites have mafic, intermediate and felsic compositions. Their geochemistry suggests that the mafic granulites are tholeiitic, whereas the intermediate and felsic granulites are calc-alkaline. Based on Green et al. (1972), Drury (1978) and Weaver and Tarney $(1980,1981)$, it is possible to conclude that, except for $\mathrm{Eu}$, the REE patterns and abundance remained unchanged in most cases, regardless of metamorphism. Thus, the more immobile elements ( $\mathrm{Mg}, \mathrm{Fe}, \mathrm{Na}, \mathrm{Ni}, \mathrm{V}, \mathrm{Zr}$ and REE) suggest the absence of a petrogenetic link between the tholeiitic and the calc-alkaline granulites. Within each group, the following features are strong indicators of cogenetic origin:

a) the degree of REE fractionation increases with decreasing $\mathrm{MgO}$ in the mafic granulites, suggesting that they might represent distinct stages of differentiation of the same parental magma, more evident in the MMgT and the LMgT, but less in the HMgT;

b) each of the calc-alkaline groups (intermediate granulites and both groups of felsic granulites) have two distinct REE patterns and, in each group, the degree of fractionation does not correlate positively with $\mathrm{SiO}_{2}$ or any other index, which, along with the similarity of REE patterns and abundance in ali groups, strongly suggest that some of the intermediate and felsic granulites may be genetically related, in its turn also true for the two groups of felsic granulites.

All JFC lithotypes, regardless of composition and structure, underwent only partial retrogressive metamorphism. Independent of the cause of dehydration (magmatic or metamorphic) of these rocks, the available data suggest that granulitization was due to metamorphism. However, it is still uncertain if anhydrous magmatism also took place, as metamorphic and magmatic processes may occur simultaneously

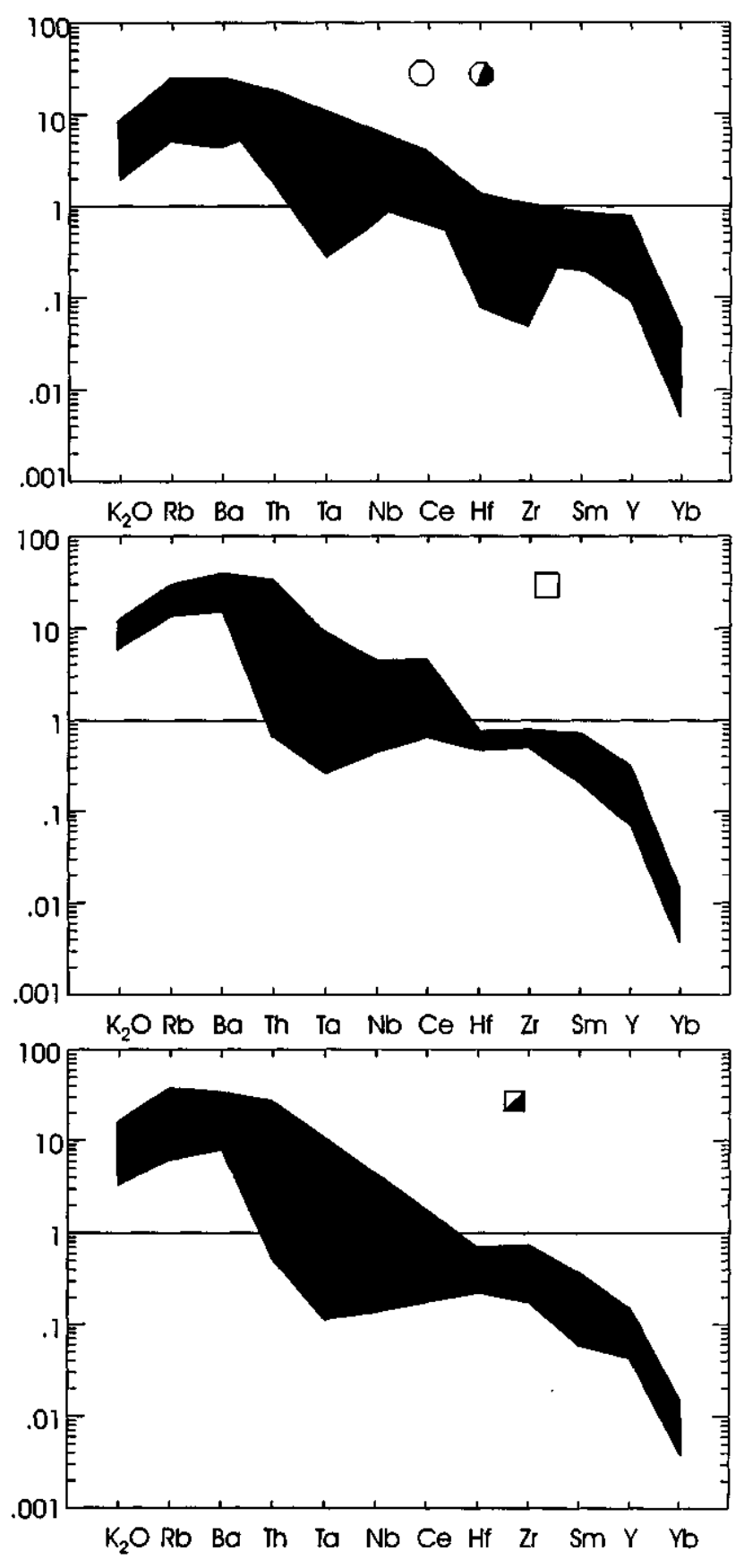

Figure 14 - ORG-normalized multi-element variation diagrams (Pearce et al 1984) for the intermediate and felsic granulites suites. Symbols as in figure 2.

Figura 14 - Diagramas de variação multi-elementar para os granulitos intermediários e ácidos, normalizados pelo ORG de Pearce et al. (1984). Símbolos como na figura 2 .

during orogenies. Two main petrogenetic hypotheses are in dispute to explain the mechanism of granulitization, i.e., partial melting (Fyfe 1973) and $\mathrm{CO}_{2}$ metamorphism (Newton et al. 1980). The former hypothesis is less probable to have taken place in the study area due to:

a) the occurrence of orthpyroxene in ali studied rocks, regardless of composition and structure;

b) if, as presumed in this study, the analyzed samples are quantitatively and qualitatively representative of the JFC, 
then the average granodioritic composition of this terrane is fairly similar to the upper average crust of Taylor (1969) and the partial melting hypothesis, that considers a compositional difference between upper (granodioritic) and lower (intermediate) continental crust, does not match to the JFC terrane, and

c) the large volume of felsic granulites of the JFC should not be considered as a residue of intra-crustal melts.

Therefore, the dehydration that caused granulitization of the JFC rocks is interpreted as most probably related to degassing of a $\mathrm{CO}_{2}$-rich fluid, that expelled and substituted
$\mathrm{H}_{2} \mathrm{O}$, as strongly indicated by the $\mathrm{CO}_{2}$-rich fluid inclusions of the JFC granulites (Nogueira 1994).

Aknowledgements Field work and chemical analyses were supported by grants of the Conselho Nacional de Desenvolvimento Científico e Tecnológico - CNPq, Fundação de Amparo à Pesquisa do Estado de São Paulo - FAPESP. B. P. Duarte is grateful to $\mathrm{CNPq}$ for its support through a research scholarship (Bolsa de Estudo). The authors are thankful to C. Valeriano, P. Tibana and A. M. Duarte for reviewing and improving the manuscript.

\section{REFERENCES}

ALMEIDA, F.F.M. de; AMARAL, G.; CORDANI, U.G.; KAWASHITA K. 1973. The Precambrian evolution of the South American Cratonic Margin South of Amazonas River. In: Nairn \& Stille, eds., The ocean basin and margins. New York, Plenum. V. 1, p. 411-446.

ANDREIS, R. R.; RIBEIRO, A.; PACIULLO, F. V.P.. 1989. Ciclos deposicionais no Proterozóico das folhas Barbacena e Divinópolis (setor sul) 1: 250.000. In: SIMP GEOL SUDESTE, 1, Rio de Janeiro, 1989. Boletim de Resumos... Rio de Janeiro, SBG-RJ-ES/SP. P. 97 - 98.

BARBOSA, A.L. \& GROSSI SAD, J.H. 1983a. Reinterpretação das Séries Juiz de Fora e Paraíba, em Minas Gerais e no Rio de Janeiro. In: SIMP. GEOL. MG. 2, Belo Horizonte, 1983. Anais... Belo Horizonte, SBG MG. p. 1-15.

BARBOSA, A.L. \& GROSSI SAD, J.H. 1983b. Petrografia dos charnockitos e rochas afins ao longo da divisa RJ/MG. In: SIMP. GEOL. MG., 2, Belo Horizonte, 1983. Anais... Belo Horizonte SBG. p. 63-74.

BARBOSA, A.L. \& GROSSI SAD, J.H. 1983c. Geoquímica e petrologia dos charnockitos e rochas afins do Complexo Juiz de Fora. RJ/MG. In: SIMP. GEOL. MG., 2, Belo Horizonte, 1983. Anais... Belo Horizonte SBG. p. 75-84.

BATCHELOR, R.A.; BOWDEN, P. 1985. Petrogenetic interpretation of granitoid rocks series using multicationic parameters. Chemical Geology, 48:43-55.

BOYNTON, W.R. 1984. Cosmochemistry of the rare earth elements meteorite studies. In: HENDERSON, P. (ed). Rare Earth Element Geochemistry, pp.63-114. Elsevier, Amsterdan.

CAMPOS NETO, M.C. \& FIGUEIREDO, M.C.H. 1992. A Orogênese Rio Doce. In: CONGR. BRAS. GEOL., 37, São Paulo, 1992. Boletim de Resumos Expandidos... SBG, 1:276-277.

CAMPOS NETO, M.C. \& FIGUEIREDO, M.C.H. 1995. The Rio Doce Orogeny, Southeastern Brazil. Journal of South American Earth Sciences, 8(2): 143-162.

CORDANI, U.G.; DELHAL, J; LEDENT, 0.1973, Orogeneses superposeés dans le Précambrien du Brésil sud-oriental (États du Rio de Janeiro et de Minas Gerais). Revista Brasileira de Geociências, 3(1): 1-22.

COX, K. G.; BELL. J. D. \& PANKHURST, R. J. 1979. The interpretation of igneous rocks. London, Allen \& Unwin. $450 \mathrm{p}$.

DELHAL, J.; LEDENT, D. \& CORDANI, U. 1969. Ages Pb/U, Sr/Rb et $\mathrm{Ar} / \mathrm{K}$ de formations métamorphiques et granitique du Sud-Est du Brésil (Etats de Rio de Janeiro et de Minas Gerais). Annales de Ia Société Géologique de Belgique,T.92: 271-283.

DRURY, S. A. 1978. REE distributions in a high-grade Archean gneiss complex in Scotland: implications for the genesis of ancient sialic crust. Precabrian Research, 7: 237-257.

DUARTE, B., P.; NOGUEIRA, J., R.; HEILBRON, M.; FIGUEIREDO, M. C., H., 1994. Geologia da região de Juiz de Fora e Matias Barbosa (MG). In: CONGR. BRÁS. GEOL. 38. Balneário de Camboriú 1994. Boletim de Resumos... Balneário de Camboriú, SBG. v. 2, p. 88-90.

EBERT, H. 1955. Pesquisas na parte sudeste do Estado de Minas Gerais. Relatório Anual do Diretor. Rio de Janeiro, DNPM/ DGM, p. 62-81.

EBERT, H. 1968. Ocorrência de fácies granulítica no sul de Minas Gerais e regiões adjacentes, em dependência da estrutura orogênica: hipóteses sobre sua origem. Anais da Academia Brasileira de Ciências, 40(Supl.):215-229.

FIGUEIREDO, M.C.H.; CAMPOS NETO, M.C. \& REGO, I.T.S.F. 1989. Geoquímica dos terrenos Juiz de Fora, Paraíba do Sul e Costeiro nos estados do Rio de Janeiro e Espírito Santo. In: WORKSHOP DE GEOQUIMICA ISOTOPICA GEOCRONOLOGIA E LITOGEOQUIMICA DAS REGIÔES SUL E SUDESTE DO BRASIL, São Paulo, 1989. Boletim de Resumos... SBGq-IG/USP, p.41-45.

FIGUEIREDO, M.C.H (in memorian) \& TEIXEIRA, W. 1996. The Mantiqueira Metamorphic Complex, eastern Minas Gerais State: preliminary geochronological and geochemical results. Anais da Academia Brasileira de Ciência, 68 (2): 223-246.
FYFE, W. S. 1973. The granulite facies, partial melting and the Archean crust. Phil. Trans. R. Soc. Lond., A273: 457-461.

GLIKSON, J. A. 1979. Primitive archean element distribution patterns: chemical evidence and geotectonic significance. Earth Planei. Sci Letters, 12: 309-320.

GREEN, T. H.; BRUNFELT, A. O. \& Heier, K. S. 1972. Rare-Earth element distribution and $\mathrm{K} / \mathrm{Rb}$ ratios in granulites, mangerites and anothosites, Lofoten-Vesteraalen, Norway. Geochimica et Cosmochimica Acta, 36: 241-257.

GROSSI SAD, J. H. \& BARBOSA, L. 1985. A origem dos charnockitos e rochas afins da região do Médio Paraíba do Sul, estado do Rio de Janeiro. Contribuições à geologia e petrologia. Boletim Especial da SBG-MG, p. 15-28.

HEILBRON, M. 1993. Evolução tectono-metamórfica da seção Bom Jardim de Minas-MG - Barra do Piraí-RJ, setor central da Faixa Ribeira. São Paulo. Tese de Doutoramento, IG - Universidade de São Paulo. 268p.

IRVINE, T.N. \& BARAGAR, W.R.A. 1971. A guide to the chemical classification of the common volcanic rocks. Canadian Journal of Earth Science, 8:523-548.

JENSEN, L. S..1976. A new cation plot for classifying subalkalic volcanic rocks. Ontario. Ontario Division of Mines, MP 66,22p.

LE MAITRE, R.W. 1989. A Classification of Igneous Rocks and Glossary of Terras. Blackwell, Oxford, $193 \mathrm{p}$

MACHADO FILHO, L; RIBEIRO, M.W.; GONZALEZ, S.R.; SCHENINI, C.A.; SANTOS NETO, A.; PALMEIRA, R.G.B.; PIRES J.L.; TEIXEIRA, W. \& CASTRO, H.E.F. de. 1983. In: Projeto RADAMBRASIL. Folhas SF-23/24, Rio de Janeiro/Vitória, 1.Geologia, Rio de Janeiro, MME, p. 27-304.

MACHADO, N.; VALLADARES, C.; HEILBRON, M.; VALERIANO, C. 1996. U-Pb geochronology of the central Ribeira belt (Brazil) and implications for the evolution of the Brazilian Orogeny. Precambrian Research 79: 347-361

MIDDLEMOST, E. A. K.. 1985. Magmas and magmatic rocks. Longman Group Ltd, Essex.

MIYASHIRO, A. 1978. Nature of alkalic volcanic rocks series. Contrib. Mineral. Petrol. 66: 91 -104.

NEWTON, R. C.; SMITH, J. V. \& WINDLEY, B. F. 1980. Carbonic metamorphism, granulites and crustal growth. Nature, 288:45-50.

NOGUEIRA, J.R.. 1994. Relações Tectono-estruturais e metamórficas entre metassedimentos e ortognaisses em fácies granulito na região a sudoeste de Juiz de Fora, Minas Gerais. Dissertação de Metrado, IG -Universidade Federal do Rio de Janeiro. 169p.

OLIVEIRA, M. A. F. 1980. Petrologia das rochas granulíticas da Faixa Paraíba do Sul, Estados do Rio de Janeiro e Minas Gerais. Tese de Livre Docência, IGCE-UNESP. 116p.

OLIVEIRA, M. A. F. 1981. Granulitos da Faixa Paraíba do Sul: caracteres geoquímicos dos piroxênios e valores geotermométricos. Rev. Bras. Geoc., II(4):222-226.

OLIVEIRA, M. A. F. 1982. Bulk geochemistry of the Paraíba do Sul Granulitic Belt. Rev. Brás. Geoc., 12(1-3):369-374.

PEARCE, J. A.. 1983. Role of the subcontinental lithosphere in magma génesis at active continental margins. In: Continental basalts and mantle xenoliths. HAWKESWORTH, C.J. \& NORRY, M. J., eds., Nantwich, Shiva, p. 230-249.

PEARCE, J. A. \& CANN, J. R.. 1973. Tectonic setting of mafic volacanic rocks determined using trace element analysis. Earth Planet. Sci. Letters, 19: 290-300.

PEARCE, J. A. \& NORRY, M., J.. 1979. Petrogenetic implications of TI, Zr, $\mathrm{Y}$ and $\mathrm{Nb}$ variations in volcanic rocks. Contrib. Mineral. Petrol, 69: $33-47$.

PEARCE, J. A.; HARRIS, N.B.W. \& TIDLE, A.G. 1984. Trace element discrimination diagramas for the tectonic interpretation of granitic rocks. Journal of Petrology, 25 (4): 956-983.

PINTO..C. P.. 1991. Programa Levantamentos Geológicos Básicos do Brasil. Lima Duarte. SF.23-X-COI V. Estado de Minas Gerais. Org. por Claiton Piva Pinto. Belo Horizonte, DNPM/CPRM. 224p. 
SHERVAIS, J. W.. 1982. Ti - V plots and the petrogenesis of modern and ophiolitic lavas. Earth Planei. Sci. Letters, 59: 101-118.

SUN, S. -S. 1980. Lead isotopic study of young volcanic rocks from mid ocean ridges and is.and ares. Phil. Trans. R. Soc. Lond., A297:409-445.

TAYLOR, S. R. 1969. Abundance of chemical elements in the continental crust: a new table. Geochimica et Cosmochimica Acta, 28:1273-1285.

THOMPSON, R. N.; MORRISON, M. A. DICHIN, A. P. \& HENDRY, G. L. 1983. Continental flood basalts... arachnids rule OK? In: Continental basalts and mantle xenoliths. HAWKESWORTH, C. J. \& NORRY, M. J., eds., Nantwich, Shiva, p. 158-185.

VALLADARES, C. S.. 1996. Evolução geológica do Complexo Paraíba do Sul no segmento central da Faixa Ribeira, com base em estudos de geoquímica e geocronologia U-Pb. Tese de Doutoramento, IG - Universidade de São Paulo. 147p
WEAVER, B. L. \& TARNEY, J. 1980. Rare earth geochemistry of Lewisian granulite-facies gneisses, northwest Scotland:implications for the petrogenesis of Archean lower continental crust. Earth Planet. Sci. Letters, 51: 279-296.

WEAVER, B. L. \& TARNEY, J. 1981. Lewisian gneiss geochemistry and Archean crustal development models. Earth Planet. Sci. Letters, 55 171-180.

Manuscrito A882

Recebido em 15 de novembro de 1996

Revisão dos autores em 15 de abril de 1997

Revisão aceita em 15 de junho de 1997 